\title{
EnerNex
}

\section{Lemnos Interoperable Security Program}

Final Scientific/Technical Report 


\section{CITATIONS}

This document was prepared by:

\section{EnerNex}

620 Mabry Hood Road, Suite 300

Knoxville, TN 37932

Principal Investigator:

Brian Smith, EnerNex

Consortium/teaming members:

\section{John Stewart, Tennessee Valley Authority \\ Ron Halbgewachs, Sandia National Laboratories \\ Adrian Chavez, Sandia National Laboratories \\ Rhett Smith, Schweitzer Engineering laboratories \\ David Teumim, Teumim Technical}

This document describes research sponsored by the United States Department of Energy (DOE) and should be cited in the literature in the following manner:

Lemnos Interoperable Security Program: Final Scientific/Technical Report. United States Department of Energy (DOE), Award Number: DE-FC26-07NT43315. 


\section{DISCLAIMER}

This report was prepared as an account of work sponsored by an agency of the United States Government. Neither the United States Government nor any agency thereof, nor any of their employees, makes any warranty, express or implied, or assumes any legal liability or responsibility for the accuracy, completeness, or usefulness of any information, apparatus, product, or process disclosed, or represents that its use would not infringe privately owned rights. Reference herein to any specific commercial product, process, or service by trade name, trademark, manufacturer, or otherwise does not necessarily constitute or imply its endorsement, recommendation, or favoring by the United States Government or any agency thereof. The views and opinions of authors expressed herein do not necessarily state or reflect those of the United States Government or any agency thereof. 


\section{EXECUTIVE SUMMARY}

The manner in which the control systems are being designed and operated in the energy sector is undergoing some of the most significant changes in history due to the evolution of technology and the increasing number of interconnections to other system. With these changes however come two significant challenges that the energy sector must face; 1 ) Cyber security is more important than ever before, and 2) Cyber security is more complicated than ever before. A key requirement in helping utilities and vendors alike in meeting these challenges is interoperability. While interoperability has been present in much of the discussions relating to technology utilized within the energy sector and especially the Smart Grid, it has been absent in the context of cyber security.

The Lemnos project addresses these challenges by focusing on the interoperability of devices utilized within utility control systems which support critical cyber security functions. In theory, interoperability is possible with many of the cyber security solutions available to utilities today. The reality is that the effort required to achieve cyber security interoperability is often a barrier for utilities. For example, consider IPSec, a widely-used Internet Protocol to define Virtual Private Networks, or " tunnels", to communicate securely through untrusted public and private networks. The IPSec protocol suite has a significant number of configuration options and encryption parameters to choose from, which must be agreed upon and adopted by both parties establishing the tunnel. The exercise in getting software or devices from different vendors to interoperate is labor intensive and requires a significant amount of security expertise by the end user. Scale this effort to a significant number of devices operating over a large geographical area and the challenge becomes so overwhelming that it often leads utilities to pursue solutions from a single vendor. These single vendor solutions may inadvertently lock utilities into proprietary and closed systems

Lemnos is built on the successes of Open PCS Security Architecture for Interoperable Design (OPSAID), a previous DOE National SCADA Test Bed (NSTB) project. It enhances security interoperability by identifying basic cyber security functions based on utility requirements and then selecting open source solutions, namely Internet Engineering Task Force (IETF) RFCs, to support these functions. Once identified, specific configuration parameters for each RFC suitable for the electric utility control system environment are identified and documented. These configuration parameters are referred to as Interoperable Configuration Profiles (ICP) and their effectiveness within the utility control systems environment is verified with comprehensive testing as the final step in the process. The project focused on development of ICPs for four security protocols (IPsec, SSH, LDAP, and Syslog) which represent fundamental building blocks which can be utilized for securing utility control systems. These ICPs are product agnostic and can be applied modularly to any device (router, substation gateway, intelligent electronic device, etc.) within the utility control system as the end user deems necessary for their unique system architecture.

The Lemnos Interoperable Security Program is a public-private partnership under the U.S. Department of Energy (DOE) Office of Electricity Delivery and Energy Reliability's Cybersecurity for Energy Delivery Systems (CEDS) program and supports The Roadmap to Secure Energy Delivery Systems. In addition to EnerNex, the core team supporting the effort includes Tennessee Valley Authority, Sandia National Laboratories, and Schweitzer Engineering Laboratories. Adding to the core team effort is collaboration from additional industry participants in the project including the Electric Power Research Institute (EPRI), Alien Vault, Cisco, Encore Networks, GarrettCom, Industrial Defender, N-Dimension Solutions, Phoenix Contact, RuggedCom, and Siemens. 


\section{CONTENTS}

1 BACKGROUND

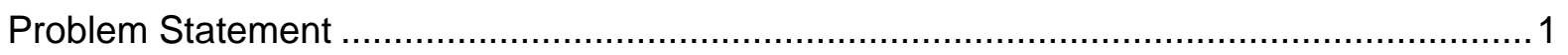

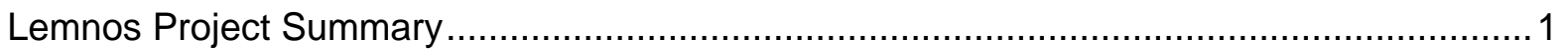

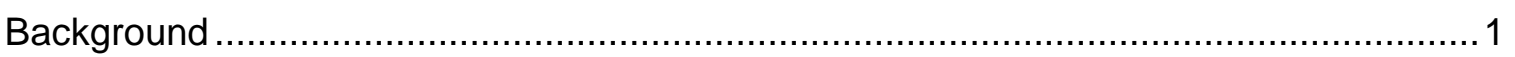

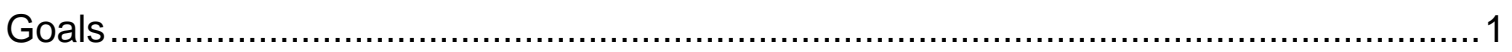

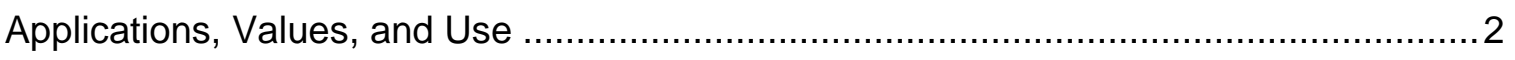

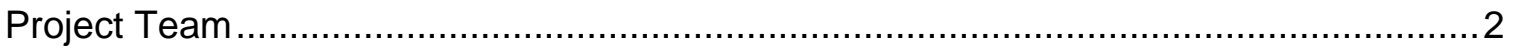

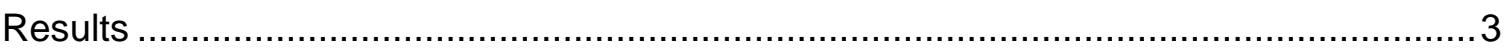

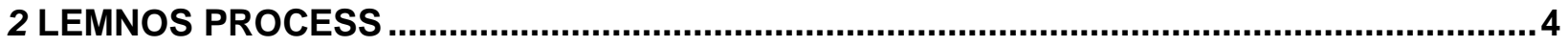

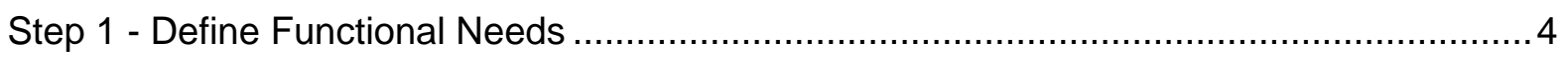

Step 2 - Identify Open Source Solution .................................................................... 5

Step 3 - Develop Interoperable Configuration Profiles.......................................................... 5

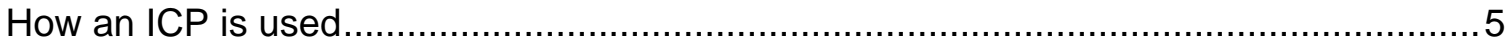

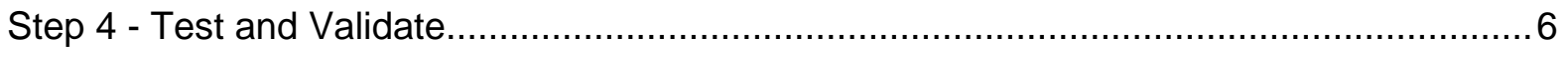

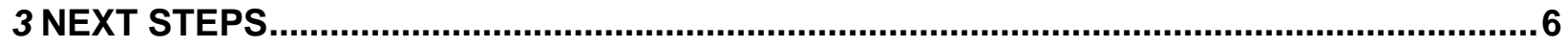

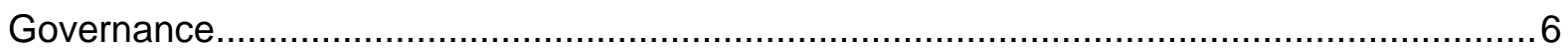

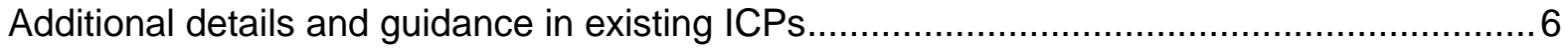

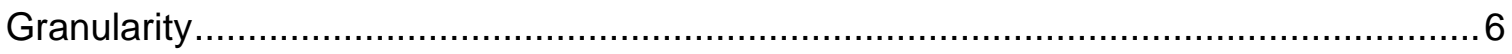

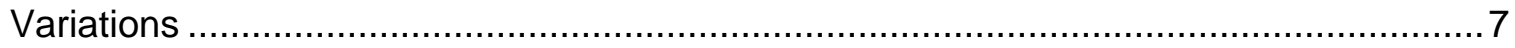

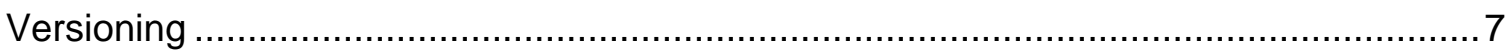

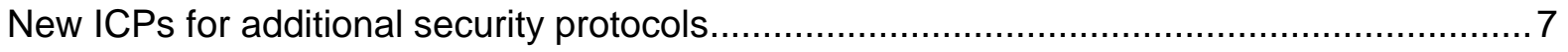

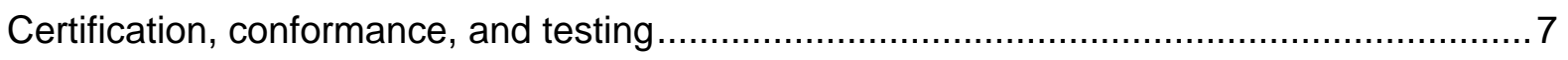

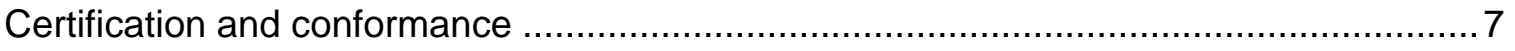

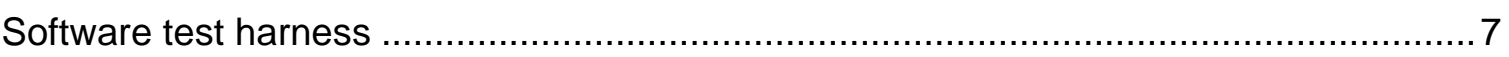

Application of ICPs beyond security devices ……....................................................

APPENDIX A： INTEROPERABLE CONFIGURATION PROFILE FOR IPSEC .................... A-1

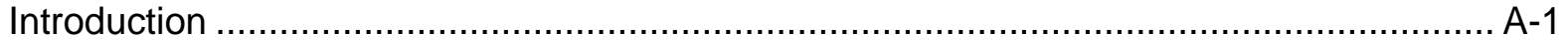

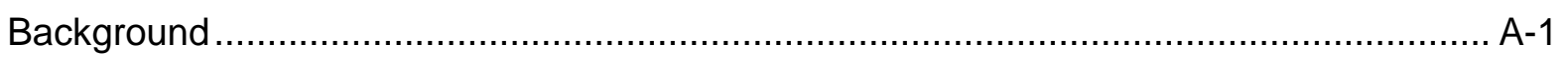

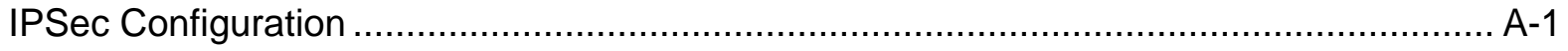

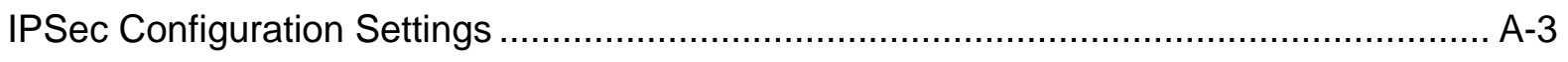

APPENDIX B: INTEROPERABLE CONFIGURATION PROFILE FOR SSH........................ B-1

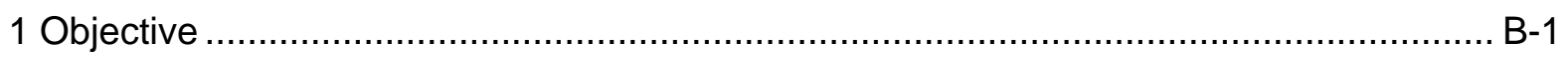

2 Scope

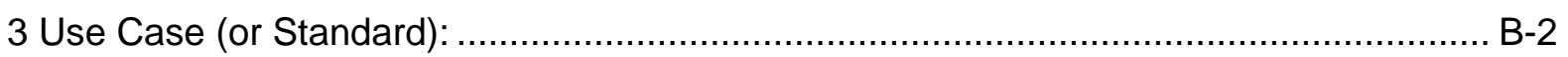

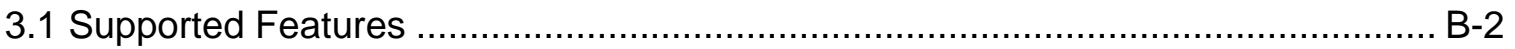

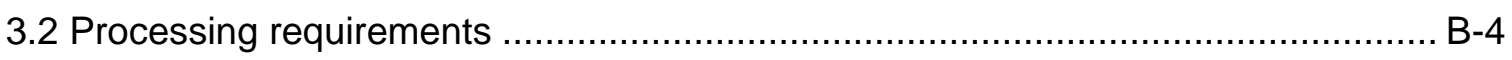

4 References: 
APPENDIX C: INTEROPERABLE CONFIGURATION PROFILE FOR LDAP .................... C-1

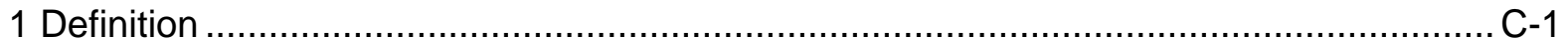

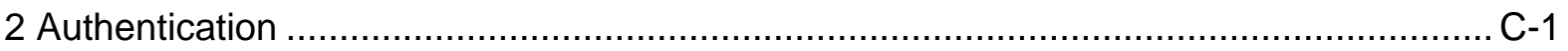

3 Authentication Implementation Overview............................................................. C-1

4 Central Authentication................................................................................ C-1

5 Additional Security Implementation Requirements ............................................. C-1

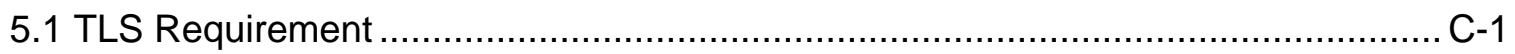

5.2 Client and Server Side Certificate ................................................................... C-2

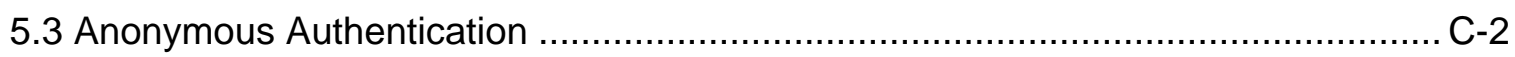

5.4 Unauthenticated Authentication .............................................................. C-2

5.5 Custom Database Schema ....................................................................... C-2

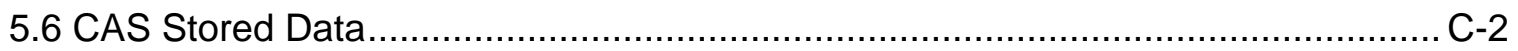

5.7 Mapping of CAS Groups to Product Setting Access ........................................... C-2

5.8 Static Database Search Parameters .......................................................... C-2

5.9 CAS Unavailability ......................................................................................

5.10 No Automatic Local Account Updates.................................................... C-2

5.11 Required TLS Algorithms ...................................................................... C-2

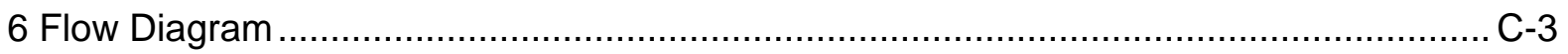

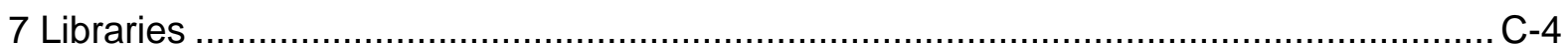

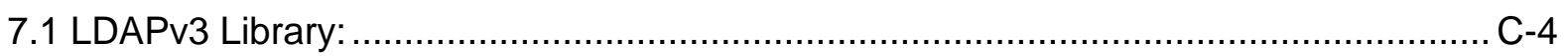

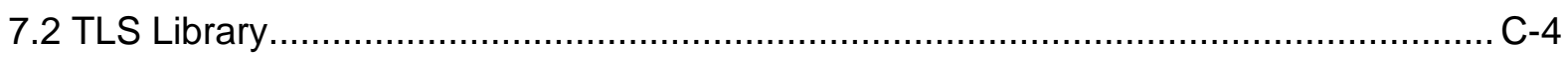

APPENDIX D: STANDARDIZATION OF SYSLOG MESSAGE....................................... D-1

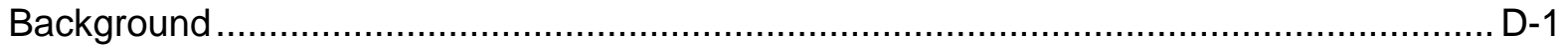

APPENDIX E: SECURITY CORE FUNCTION AND DEFINITION $\ldots \ldots \ldots \ldots \ldots \ldots \ldots \ldots \ldots \ldots \ldots . . . \ldots \ldots \ldots$

Security core functional and nonfunctional Requirements ......................................... E-1

Functional/Nonfunctional Mapping .................................................................... E-1

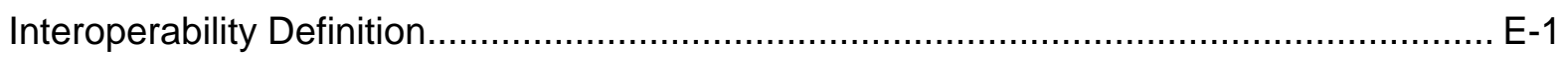

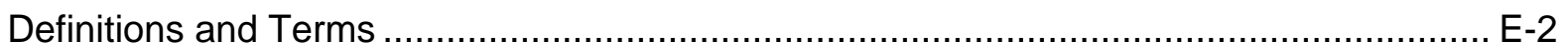

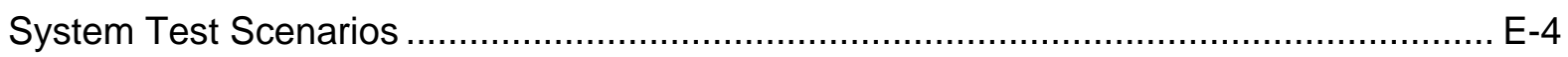

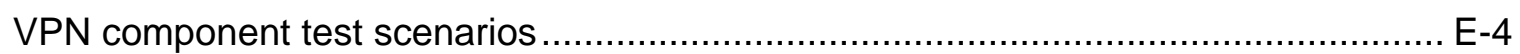

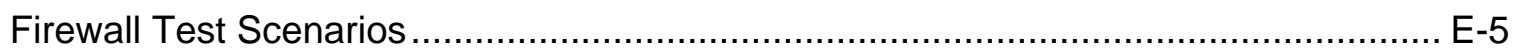

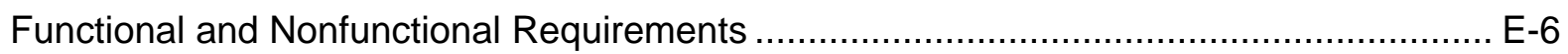

APPENDIX F: SECURITY FUNCTIONALITY SELECTION ..........................................F-1

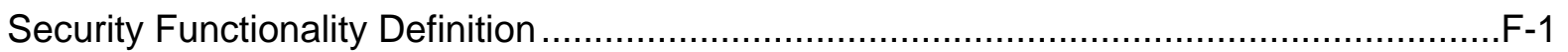

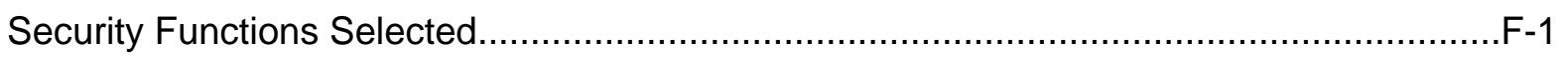

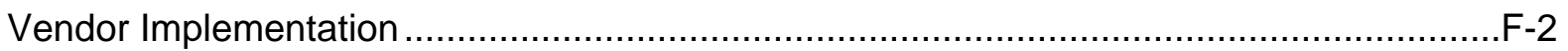

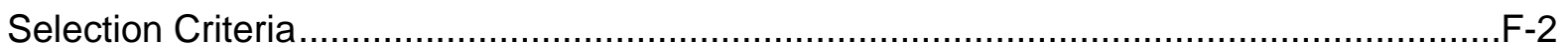

APPENDIX G： TVA TESTING (JULY 2009).......................................................................

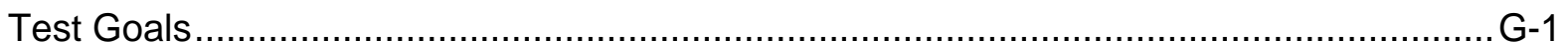

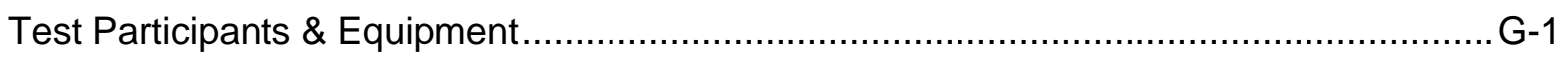




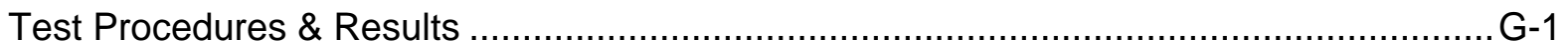

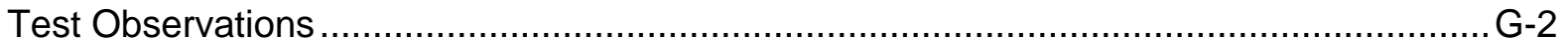

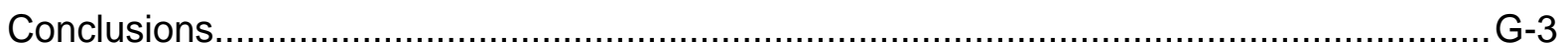

APPENDIX H: EPRI TESTING (2011) ..................................................................... H-1

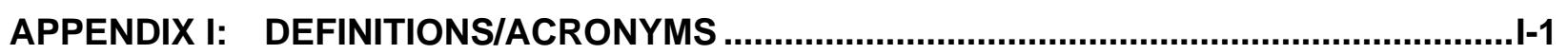




\section{BACKGROUND}

\section{Problem Statement}

The manner in which the control systems are being designed and operated in the energy sector is undergoing some of the most significant changes in history due to the evolution of technology and the increasing number of interconnections to other systems. With these changes however, come two significant challenges that the energy sector must face; 1 ) Cyber security is more important than ever before, and 2) Cyber security is more complicated than ever before. A key requirement in helping utilities and vendors alike in meeting these challenges is interoperability. While interoperability has been present in much of the discussions relating to technology utilized within the energy sector and especially the Smart Grid, it has been absent in the context of cyber security.

\section{Lemnos Project Summary}

\section{Background}

Lemnos is a multiyear, public-private partnership effort funded under the DOE Office of Electricity Delivery and Energy Reliability's Cybersecurity for Energy Delivery Systems (CEDS) program which supports The Roadmap to Secure Energy Delivery Systems.

\section{Goals}

The goal of the Lemnos project is to address the interoperability of devices utilized within utility control systems which support critical cyber security functions. In theory, interoperability is possible with many of the cyber security solutions available to utilities today. The reality is that the effort required to achieve cyber security interoperability is often a barrier for utilities. For example, consider IPSec, a widely-used Internet Protocol to define Virtual Private Networks, or "tunnels", to communicate securely through untrusted public and private networks. The IPSec protocol suite has a significant number of configuration options and encryption parameters to choose from, which must be agreed upon and adopted by both parties establishing the tunnel. The exercise in getting software or devices from different vendors to interoperate is labor intensive and requires a significant amount of security expertise by the end user. Scale this effort to a significant number of devices operating over a large geographical area and the challenge becomes so overwhelming that it often leads utilities to pursue solutions from a single vendor. These single vendor solutions may inadvertently lock utilities into proprietary and closed systems. 


\section{Applications, Values, and Use}

Interoperable Configuration Profiles are developed to describe a specific instantiation of a particular security related protocol. The hypothesis of the DOE Lemnos work is that if a vendor independently implements a security function based on an Interoperable Configuration Profile and tests it against the reference implementation developed and maintained by Sandia National Laboratories, that product should interoperate with any other product which has done the same. This process alone is not sufficient however to ensure the effectiveness of the Interoperable Configuration Profiles and therefore additional testing must be conducted and corresponding conformity goals must be developed. Once adopted, the Interoperable Configuration Profiles will make it easier to procure and implement secure systems, reducing the cost of integration, facilitating competition among the vendor community to reduce prices, and minimizing the cost of configuring and maintaining devices supporting cyber security functions over their lifetime. This EPRI project is providing valuable feedback to the DOE Lemnos team as well as OpenSG on the effectiveness and usability of the Interoperable Configuration Profiles which in turn, is condensing the traditional development cycle for specifications of this type.

\section{Project Team}

- Project Partners

o EnerNex (Prime DOE Contractor)

o Tennessee Valley Authority

- Sandia National Laboratories

- Schweitzer Engineering Laboratories (SEL)

- Participating R\&D Organizations

o Electric Power Research Institute (EPRI)

- Participating Vendors
o Alien Vault
o Cisco
o Encore Networks
o GarrettCom
o Industrial Defender
o n-dimension
o RuggedCom
o Phoenix Contact
o Siemens 


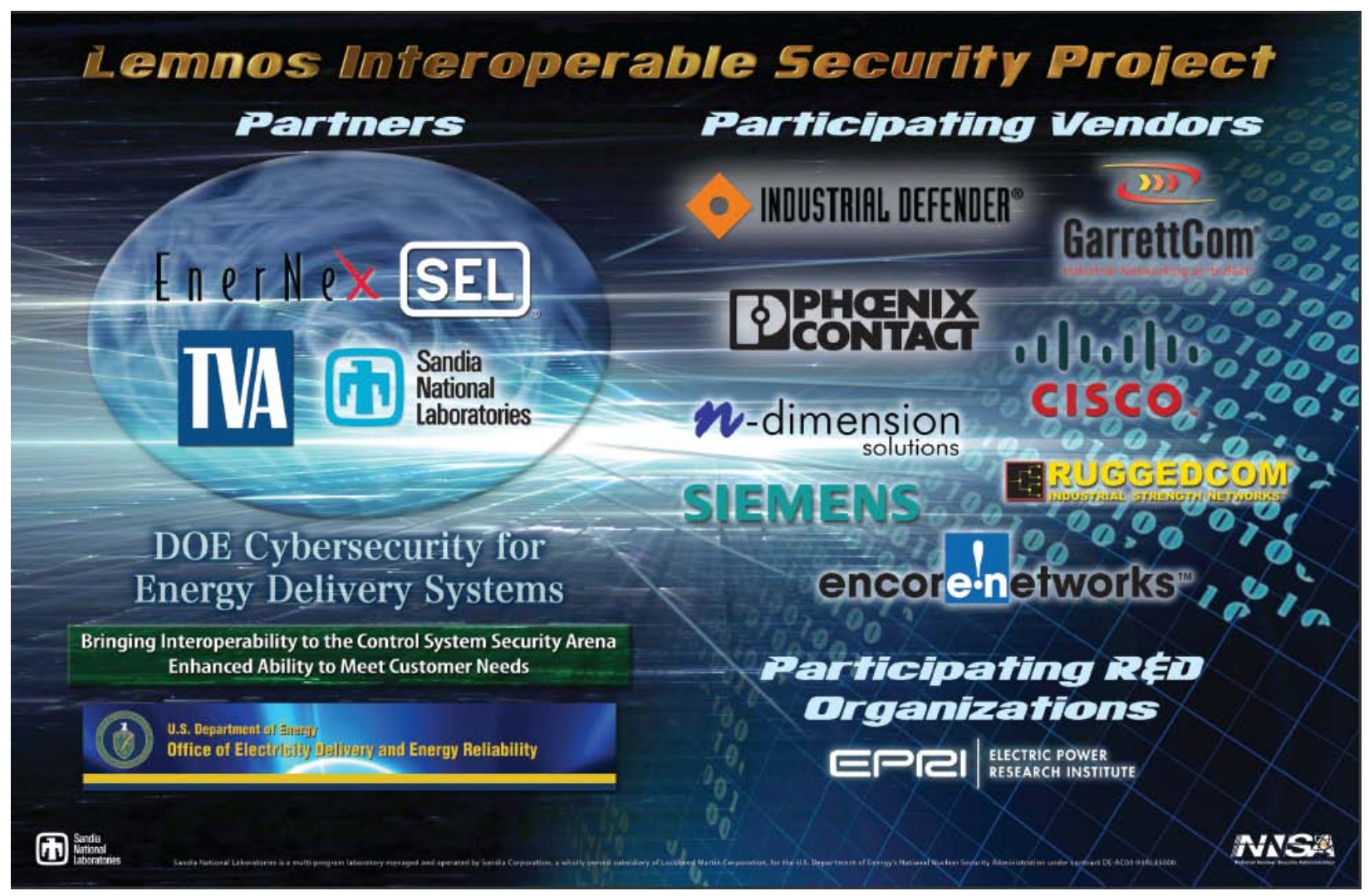

Figure 1-1

Lemnos Project Team

\section{Results}

The Lemnos project has produced guidance for four security protocols as shown in Table 1-1.

\begin{tabular}{|l|l|l|}
\hline $\begin{array}{l}\text { Functional Requirement } \\
\text { Secure communications channel }\end{array}$ & $\begin{array}{l}\text { Component } \\
\text { Virtual Private }\end{array}$ & $\begin{array}{l}\text { IPsec } \\
\text { (RFC 4301) }\end{array}$ \\
\hline Notification, Traceability, Trouble Shooting & Audit Log & $\begin{array}{l}\text { Syslog } \\
\text { (RFC 5424/3164) }\end{array}$ \\
\hline Centralized Authentication & Authentication & $\begin{array}{l}\text { LDAP } \\
\text { (RFC 4510) }\end{array}$ \\
\hline Secure Remote Access & $\begin{array}{l}\text { Virtual Private } \\
\text { Network } \\
\text { (Application }\end{array}$ & TLS/SSH \\
\hline Layer) & \\
\hline
\end{tabular}

Table 1-1

Security Protocols Utilized in Lemnos Project 
Appendices A, B, and C of this document outline the technical details contained within the ICPs for the IPSec, SSH, and LDAP protocols respectively.

The scope of this initial effort only covered the use of Syslog and did not prescribe any specific configuration parameters therefore no formal ICP document was developed. Appendix D outlines the results of the work focused on the standardization of the Syslog message Payload.

\section{LEMNOS PROCESS}

The ultimate output of the Lemnos work are artifacts referred to as Interoperable Configuration Profiles however the method to produce these profiles is just as important. The Lemnos work is formed around asset owner functional requirements relating to cyber security which means it is as much about the process as it is about the artifacts which it creates. By focusing the effort in this fashion, the process provides a linkage between these asset owner requirements and the technical solutions deployed to meet them by developing and publishing Interoperable Configuration Profiles for security products. Targeted at security products utilized within control systems deployed throughout the electric utility sector, the approach taken by Lemnos to create the Interoperable Configuration Profiles covers four basic steps as shown in Figure 2-1 that follows.

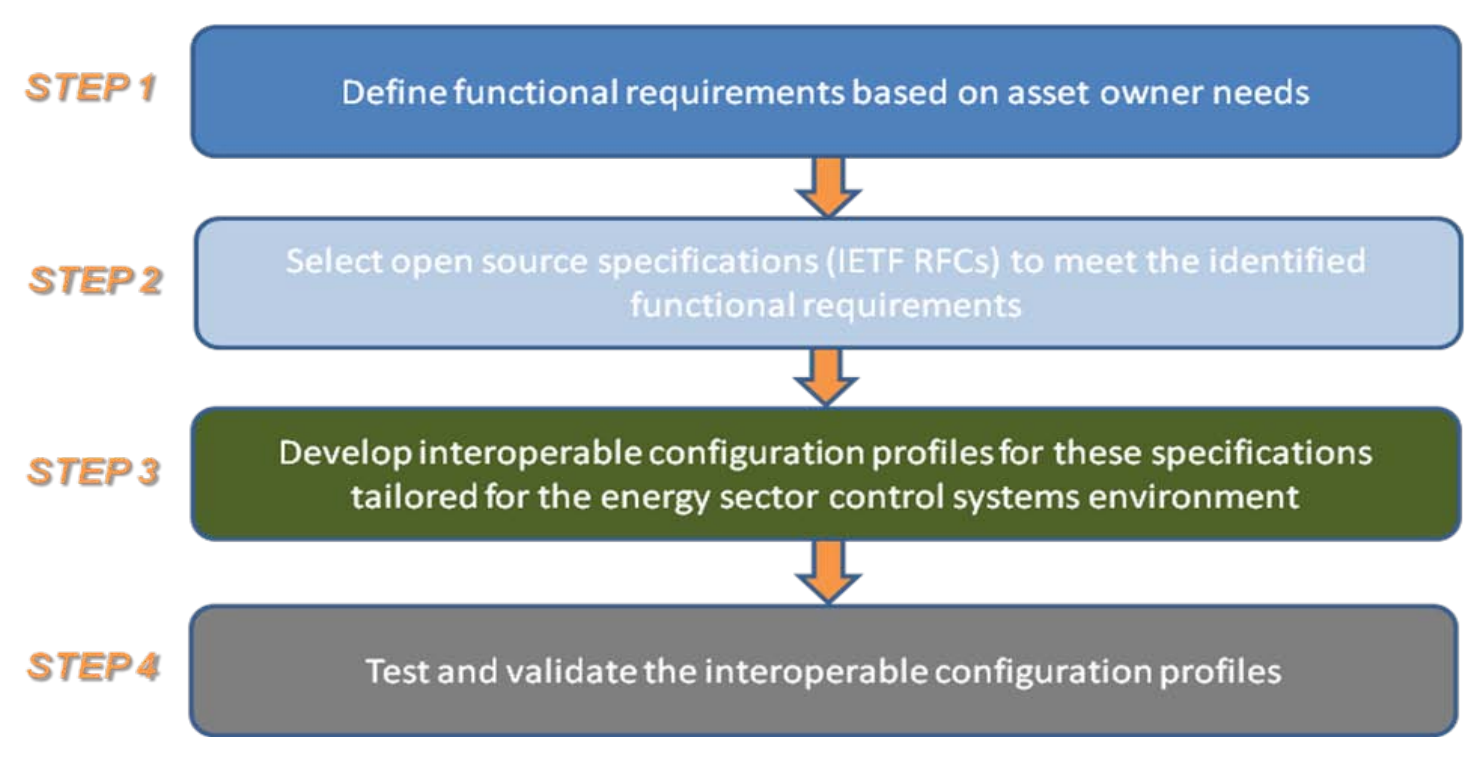

Figure 2-1

Lemnos Process

\section{Step 1 - Define Functional Needs}

The first step of the Lemnos process defines the problem in terms of core security functional requirements which may be needed by asset owners in various parts of their control system design. In short, we need to know what the problem is before jumping head first into solving it and risk missing the point altogether. It sounds simple, but it's perhaps the most important step of the entire process. This step involves asking a set of binary questions. The subject either does something (performs a specific function) or it does not and there is no measurement involved. At the end of the step is a list of the core security functional 
requirements (i.e. the "problem") which can then be easily prioritized. For the initial Lemnos effort, numerous core functions were identified based on asset owner input as shown in Table 2-1 that follows.

Core Security Functional Requirement

Secure communications channel

Filter illegal network traffic

Notification, traceability, and troubleshooting

Cryptography and password management

Detect malicious activity by monitoring network traffic

Monitor and analyze system processes

Table 2-1

Core Security Functional Requirements

Appendix $E$ of this report outlines the project team's efforts in this area.

\section{Step 2 - Identify Open Source Solution}

The next step of the process takes each selected core security functional requirement and pairs it with a solution. For these solutions in the past, vendors may have developed their own proprietary solution or perhaps implemented a little known solution which did not effectively match the needs of the control systems environment. With Lemnos, the philosophy is to select the most commonly used, well-proven, open source solution for each requirement from within the IETF (Internet Engineering Task Force) library of RFC's (Request For Comments). Appendix F of this report outlines the project team's efforts in this area.

\section{Step 3 - Develop Interoperable Configuration Profiles}

The selection of one or more IETF RFC'S is just a starting point for many more decisions to come in the development of the Interoperable Configuration Profiles. Each RFC contains a myriad of choices that have to be made for implementation. Choices which cannot be made in a vacuum but which must be made with the supported utility applications and the underlying control system architecture in mind. By doing so, each Interoperable Configuration Profile provides both a definition of cyber security interoperability and a minimum documented level of security for the targeted protocol(s).

Appendices A, B, and C of this document outline the technical details contained within the ICPs for the IPSec, SSH, and LDAP protocols respectively.

\section{How an ICP is used}

While the primary use of the Interoperable Configuration Profiles is to define interoperability for various IP protocols utilized for cyber security, they can also be utilized to communicate requirements and compare devices. The Lemnos work does not create a "one size fits all" structure which end users should require and vendors should implement in its entirety on each and every system device. Instead, the multiple Interoperable Configuration Profiles create a modular approach which allows users to require and vendors to support any combination of the Interoperable Configuration Profiles. What it means to end users and vendors is that as more Interoperable Configuration Profiles are created, they will have more choices. This modularity allows for the appropriate set of functionality to be applied on a case by case basis at the device level based on the device's role in the overall system. Host device or network node, Interoperable Configuration Profiles can assist end users in communicating device requirements and 
vendors in communicating device functionality for any networked device which must support a core security functional requirement.

\section{Step 4 - Test and Validate}

While testing is part of the standard development process for any device, the testing involved in the Lemnos process is aimed at two specific areas:

1. Validating the effectiveness of the Interoperable Configuration Profiles to clearly describe the parameters necessary to establish interoperability between two devices supporting covered security functionality.

2. Assess any potential impacts to the control systems environment from the addition of the specified technology. This is done by deploying this new technology into control system architectures and run simulated real world experiments. Various sites are used to test the Interoperable Configuration Profiles in differing control system architectures and under different data loads and different data protocols. This allows for analysis of the system impact as well as the impact on the personnel that will be tasked with deploying and maintaining it.

Appendices $\mathrm{G}$ and $\mathrm{H}$ of this report outline results of testing at TVA and EPRI during the course of this project.

\section{NEXT STEPS}

As noted previously, the Lemnos project is a public-private partnership funded primarily by the U.S. Department of Energy. DOE funding in support of the current Lemnos effort ends Q1 of 2012. As such, the goals of the Lemnos project to date have been targeted at commercialization of a limited set of security protocols at a fixed point in time. While the project has achieved the specific goals for which it set out, the effort as it stands cannot sustain long term value to utilities or vendors without continuing and expanding. The following outline specific areas which have been identified by the stakeholders as enhancing the value and longevity of the Lemnos work.

\section{Governance}

For long term success of the Lemnos work, a basic governance structure needs to be created. This would address items such as:

- Processes for creating, revising, reviewing, and commenting on ICPS

- Maintenance and upkeep of the core security functions list

- Accessibility and repository of documents

\section{Additional details and guidance in existing ICPS}

For the three ICPs which have been developed to date, potential follow on efforts identified as high value include:

\section{Granularity}

There is no complete list of parameters that can be documented to guarantee interoperability and the current Lemnos ICPs have been developed to address the major components of the IETF RFCs. Each time the existing ICPs are vetted and tested by the vendors, additional details needed within the documents are identified to further define interoperability. The more specific the details are within and $I C P$, the lesser the chance that two products may not interoperate in the desired fashion. 


\section{Variations}

While the current concept of the Lemnos ICPS is that a single ICP would apply to any instance of the security protocol in use within the utility's control system,the reality is that as the use of the ICPS is expanded, it may highlight the need for variations of an ICP for a single security protocol.

\section{Versioning}

If the ICPs are to become an effective tool which utilities and vendors use in the foundation of their security architecture, they must be kept current. The original scope of the Lemnos effort was for finite duration and therefore did not address a process by which the ICPs would be updated to evolve with the RFCs as well as vulnerabilities. One specific challenge that must be addressed from a governance point of view is going to be a potential lack of backwards compatibility if an ICP is updated to no longer allow the use of a component, such as a cryptographic algorithm or hash, which has been deprecated. This issue would also influence any efforts to establish testing and conformance guidance.

\section{New ICPs for additional security protocols}

There are many variations from utility to utility in the manner by which control systems are designed and built. Within these architectures, the identified Core Security Functions are intended to define the utility's needs based on functional requirements which do not change as rapidly as technology. To be effective, the Lemnos work must provide choices for the identified Core Security Functions where they are warranted. For example, the current ICP for LDAP supports a utility's need for centralized authentication. LDAP is not the only viable solution for centralized authentication and some utilities may desire to utilize RADIUS in lieu of or in addition to LDAP.

\section{Certification, conformance, and testing}

Utilities need assurance that when they purchase components and systems, the underlying security mechanisms employed will be compatible and interoperable. While the ICP documents serve as a good foundation for this purpose, it still requires a significant effort on the part of the utility to validate a vendor's claim. To address this need, two specific areas have been identified as follows:

\section{Certification and conformance}

Establishing a certification and conformance framework is a critical element to any long term Lemnos effort. Any effort in this area would need to be aligned with the guidance being developed by the Smart Grid Interoperability Panel (SGIP) and the UCAlug's OpenSG Technical Committee. A first step in this area would involve developing test procedures for each Lemnos ICP. This exercise may also drive the need for revisions to the ICP documents themselves.

\section{Software test harness}

To date, a vendor wishing to test a product's ability to support a Lemnos ICP required testing with another vendor's product or the Sandia National Labs OPSAID reference implementation. This has been a manageable task for the small number of vendors and products which have been part of the Lemnos effort to date. If the effort continues to grow however, the effectiveness of this approach would be limited and potentially negatively impact the overall success of the Lemnos efforts.

A software test harness supporting the Lemnos ICPs would allow for the independent and automated tests of a specific ICP implementation within individual products. This would be driven by test procedures identified in the previous section and enhance both the vendor's ability to provide products supporting the Lemnos efforts as well as the utility's ability to independently verify a product meets its requirements.

\section{Application of ICPs beyond security devices}

In theory, ICPs have been created to be product agnostic. Their application and testing within the Lemnos project to date however has been limited to security devices which are typically utilized to secure systems at their perimeter. Feedback from utility stakeholders indicates that end-to-end security is the desired 
state which means that some security functions need to be extended down to the end devices such as protective relays, digital fault recorders, remote terminal units, or other Intelligent Electronic Devices (IED). This effort would involve identifying a select number of end devices and working with the corresponding vendors to implement the functionality outlined in the relevant ICP. This in turn may drive the need for more granularity or variations of ICPs for a given security protocol. 


\section{APPENDIX A: INTEROPERABLE CONFIGURATION PROFILE FOR IPSEC}

Following is the contents of the most recent Interoperable Configuration Profile for IPSec as of the date of this publication.

\section{Introduction}

The effort required to achieve cyber security interoperability is often a barrier for utilities implementing technologies. Let's consider IP Security Protocol (IPSec) as an example, IPSec is a widely-used Internet Protocol to define Virtual Private Networks (VPN), or "tunnels", to communicate securely through untrusted public and private networks. The IPSec protocol, defined by the Internet Engineering Task Force (IETF) ${ }^{1}$, has a significant number of configuration options and encryption parameters to choose from. The IETF has many Request for Comments (RFC) for the IPSec protocol. How would a utility decide on which IPSec configuration setting to use for a secure tunnel? The configuration options must be agreed upon and adopted by both parties prior to establishing the tunnel. The process of setting up a secure tunnel and getting different vendors to interoperate securely is labor intensive and may require a significant amount of security expertise by the end user. This whitepaper will document a secure configuration tunnel profile that will interoperate with different vendors.

\section{Background}

To obtain interoperability between proprietary security appliance units, one or both vendors must now write cumbersome "translation code." If one party changes something, the translation code "breaks." The Lemnos project is developing and testing a framework that uses widely available security functions and protocols, like IPsec, to exchange security log messages. Using this model, security appliances from two or more different vendors can clearly and securely exchange information, helping to better protect the total system.

\section{IPSec Configuration}

As a utility would start a process to setup a VPN, there are a myriad a choices for the configuration. Initially, using the diagram from a popular IPSec tutorial on the Web, a nice decision tree exists to assist a utility in deciding basic VPN configuration parameters. See Figure A-1, The IPsec Transform Creation Decision Tree. A good reference for an IPSec tutorial can be found at this link:

\section{http://www.eetimes.com/design/communications-design/4014693/IPsec-a-Tutorial-Part-VI}

With the Lemnos initial review, the team made the selections and tested the configurations with multiple vendors to insure they were interoperable and secure. For instance, the following choices are made from the flowchart by the Lemnos team:

- Use ESP (Encapsulating Security Payload)

- Use TUNNEL mode (vs transport)

- Use a HMAC (Hashed Message Authentication Code)- needed for authentication and integrity. Use either MD5 or SHA1 (Secure Hash Algorithm 1)

\footnotetext{
${ }^{1}$ The EITF IPSec working group will develop mechanisms to protect client protocols of IP. A security protocol in the network layer will be developed to provide cryptographic security services that will flexibly support combinations of authentication, integrity, access control, and confidentiality.
} 


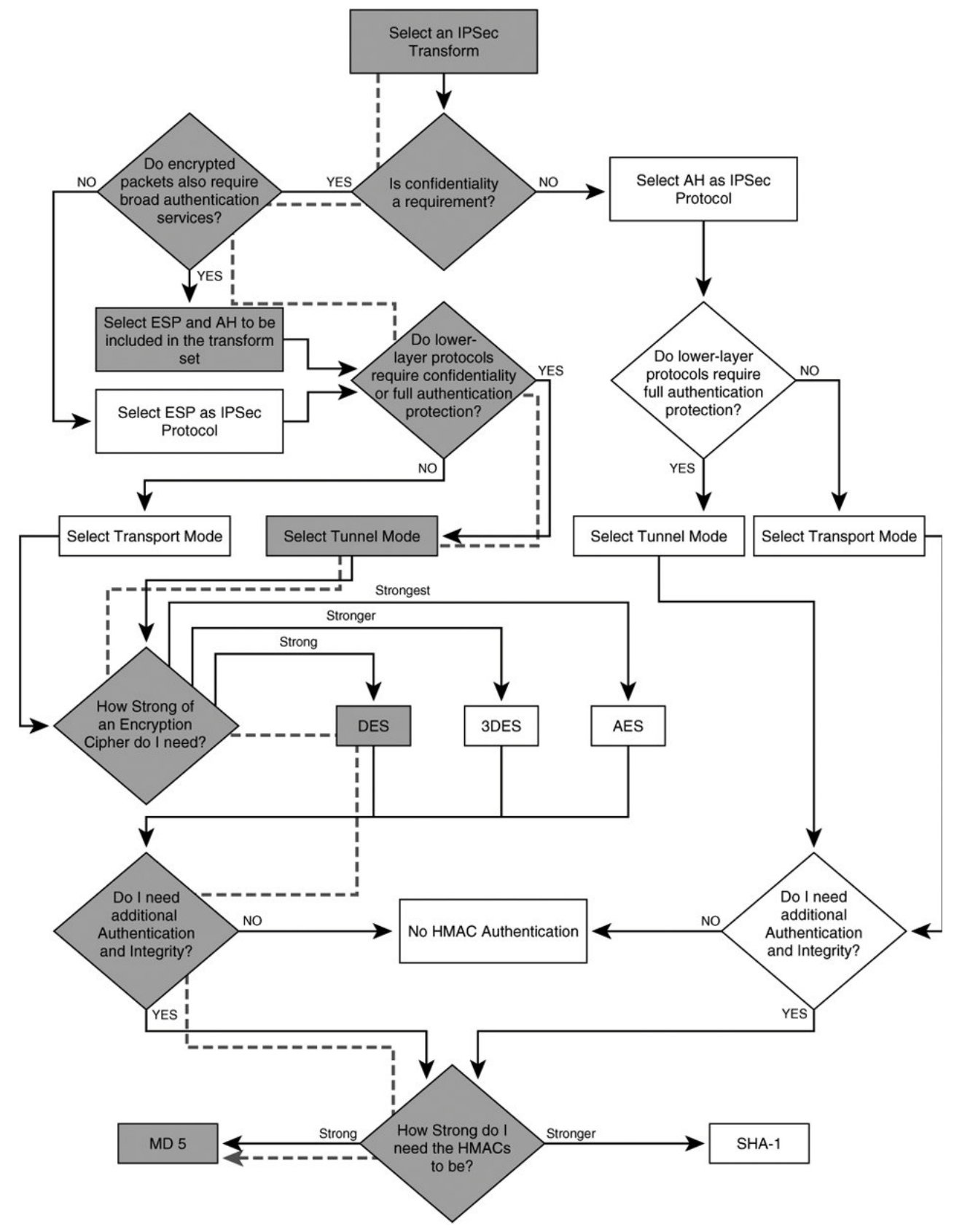

Figure A-1

The IPSec Transform Creation Decision Tree ${ }^{2}$

In a similar fashion, the Lemnos team reviewed the various IPSec RFCs, making secure interoperable choices and, very importantly, testing these choices with the variety of software that vendors on the team are using. Lemnos partners and participating vendors were using the following software at the time of testing:

\section{- Strongswan on Linux platform}

${ }^{2}$ IPSec, a Tutorial-Part VI, Commsdesign, James Henry Carmouche Dec 28, 2006 (2:00 PM),

URL:http://www.commsdesign.com/showArticle.jhtml?articleID=196800024, Reproduced from the book IPsec Virtual Public Network

Fundamentals. Copyright [2006], Cisco Systems, Inc. 
- Proprietary Stack on Wind River RTOS

- Window XP embedded IPSec stack

\section{IPSec Configuration Settings}

Basic configuration decisions included:

- Using ESP (Encapsulating Security Payload)

- Using TUNNEL mode

- Using HMAC for authentication and integrity

- Using IKE Version 1 (moving to IKE Version 2 in future)

- Using DH-5 (Diffie-Hellman Group 5)

The specific configuration parameters for configuration the IPSec VPN tunnel are as follows:

- ike_life: 28,800 s; (28,800 seconds life for key until exchange)

- ipsec_life: 3600s; ( time till key re-negotiation)

- $\quad$ rekey_margin: 540s; (default value ?)

- rekey_fuzz: $100 \%$; (default value ?)

- $\quad$ keyingtries: 3; (renegotiate keys 3 times)

- dpd_action: restart; (dead peer detection action)

- dpd_delay: 60s; (dead peer detection time "hello" interval in seconds)

- dpd_timeout: 150s; (dead peer detection time timeout interval in seconds)

- policy: PSK+ENCRYPT+TUNNEL+PFS+UP;

- Use PFS (perfect forward secrecy ); for enhanced key exchange security (Use DH5 with PFS)

The following is the Required, Recommended, and Deprecated list of Cryptographic Algorithms from the reference software configuration File

- 000 List of registered IKE 1 Encryption Algorithms:

o 000 \#7 OAKLEY_AES_CBC, blocksize: 128, keylen: 128(Required)

o 000 OAKLEY_AES_CBC,blocksize:128, keylen: 192 or 256 (Recommended)

- 000 List of registered IKE Hash Algorithms:

o 000 \#1 OAKLEY_MD5, hashsize: 128 (Required)

0000 OAKLEY_SHA1, hashsize 128 (Required)

o 000 \#4 OAKLEY_SHA2_256, hashsize: 256 (Recommended)

- 000 List of registered IKE DH Groups:

o 000 \#2 OAKLEY_GROUP_MODP1024, groupsize: 1024 (Deprecated )

o 000 \#5 OAKLEY_GROUP_MODP1536, groupsize: 1536 (Required)

- 000 List of registered ESP Encryption Algorithms:

o 000 \#12 ESP_AES, blocksize: 8, keylen: 128-256

- 000 List of registered ESP Authentication Algorithms:

o 000 \#1 AUTH_ALGORITHM_HMAC_MD5, keylen: 128-128(Required)

$0 \quad 000$ AUTH_ALGORITHM_SHA1, Keylen 128-128 (Required)

o 000 \#5 AUTH_ALGORITHM_HMAC_SHA2_256, keylen: 256-256 (Recommended) 



\section{APPENDIX B: INTEROPERABLE CONFIGURATION PROFILE FOR SSH}

Following is the contents of the most recent Interoperable Configuration Profile for SSH as of the date of this publication.

\section{Objective}

The energy sector is facing two major issues in securing control systems; 1 ) Cyber security is more important than ever before, and 2) Cyber security is more complicated than ever before. A key requirement for utilities and vendors alike in addressing these issues is interoperability. This paper continues the Lemnos Interoperability Testing Project, a multiyear DOE NSTB (National SCADA Test Bed) effort highlighting a security interoperability framework for communications supporting the energy sector. Current partners in the Lemnos project include EPRI, Cisco, EnerNex, Tennessee Valley Authority, GarrettCom, RuggedCom, N-Dimension, Sandia National Laboratories, and Schweitzer Engineering Laboratories. Lemnos is built on the successes of Open PCS Security Architecture for Interoperable Design (OPSAID), a previous NSTB project. IPSec and Syslog are used in Lemnos for interoperable communication between security devices, and is expanding to use other popular Internet Protocols like SSH. Lemnos partners and participating vendors produce Interoperable Configuration Profiles (ICPS) by consensus and verify the effectiveness of these profiles with comprehensive testing. This work is helping to foster the partnership between utilities and vendors by helping the two parties to clearly communicate user needs, product features, and configuration parameters relating to cyber security and interoperability functions.

\section{Scope}

For the SSH interoperability testing, a test network was created in a laboratory environment to examine the ICP. Sandia National Laboratory (SNL) created a "reference" server on the network with the SSH daemon configured according to the ICP specifications. The reference server is used to form the baseline configuration and to test client interaction with the daemon process. The participating vendors then configure the SSH daemon on their platform in accordance with the ICP.

The SSH ICP is designed to allow engineering access to remote locations in a secure, compliant, and vendor-neutral manner. This is accomplished by implementing the ICP on the remote daemon (server service) in a standardized and tested configuration allowing utilities to choose from multiple vendors as they implement smart grid technologies.

Previously, a utility operator needing to interact with substation equipment remotely was forced to use insecure protocols such as telnet, FTP, or an insecure proprietary protocol. Figure B-2 displays an example utility implementation utilizing the SSH ICP. A control center operator is able to securely connect to a remote vendor device, presumably in a substation, via SSH. 


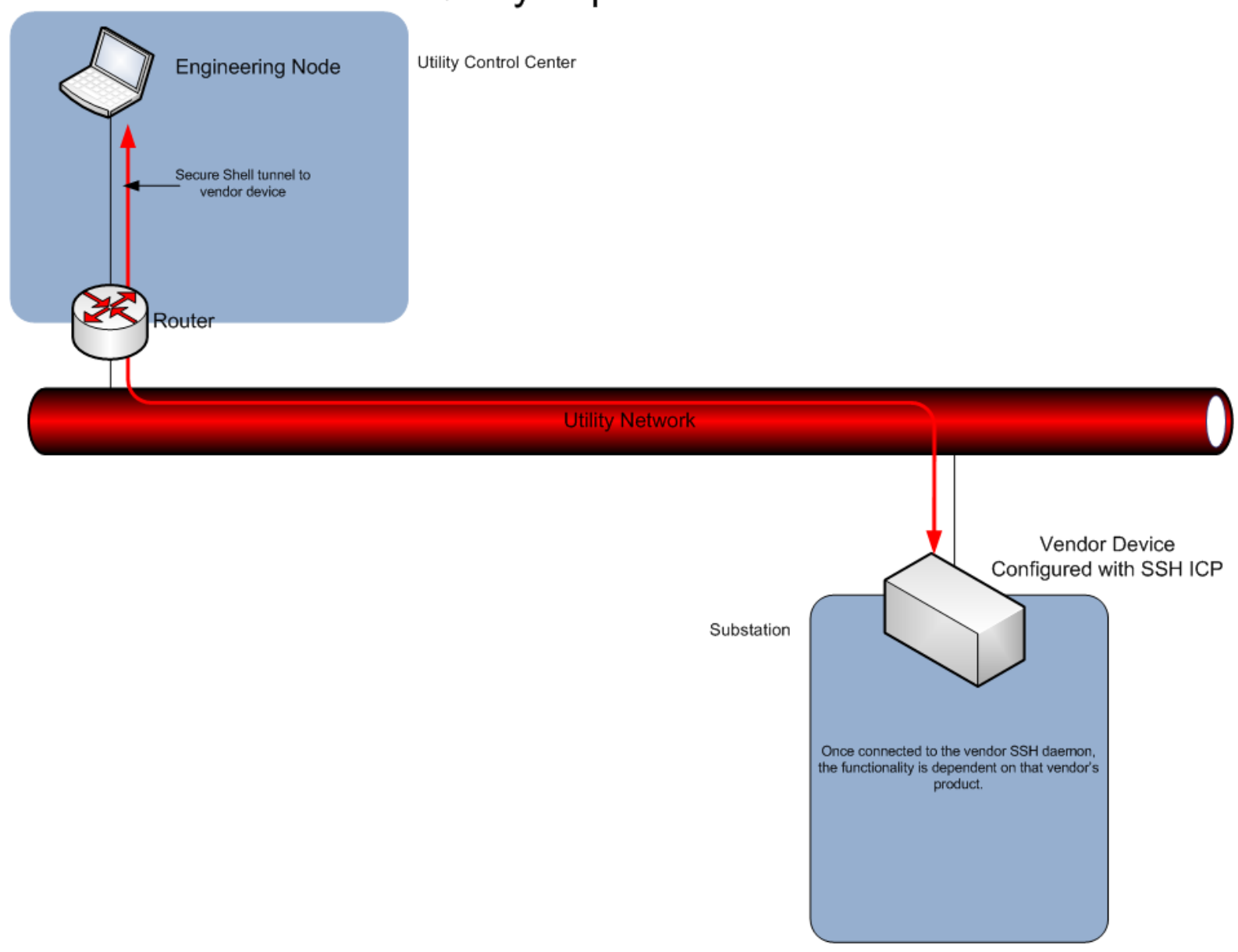

Figure B-2

SSH ICP Utility Implementation

\section{Use Case (or Standard):}

The TCP SSH implementation shall conform to all requirements in the RFCs listed in Section 2. All required options shall be accepted by the implementation. Optional parameters shall be supported as listed below.

\subsection{Supported Features}

\subsubsection{SSH Version}

The Lemnos team recommends supporting only the most recent secure version, currently version 2 , of $\mathrm{SSH}$. Any SSH daemon (server service) instance shall be configured to disable/disallow support for previous vulnerable versions. All connections from earlier versions of SSH implementations shall be rejected.

The TCP SSH implementation shall report a software version (RFC4253 Section 4.2) where <version> shall be replaced with a version string unique to each release of the implementation. This version string shall be used to identify feature differences in future releases. The implementation shall not obfuscate or mislead the version string. 


\subsubsection{Ciphers}

In accordance with NIST SP-131a ${ }^{3}$, select ciphers are recommended in order to maintain compliance with NIST-IR 7628. It should be noted that the goal of the Lemnos ICP is not to achieve compliance; rather, the goal is to prevent non-compliance. The SSH implementation shall support the following encryption ciphers:

- AES 256 CTR

- AES_256_CBC

- AES_128_CTR

- $A E S \_128$-CBC

The SSH implementation shall NOT support ciphers that are deprecated or are scheduled to be deprecated including:

- Blowfish

- DES

- 3DES (two-key)

In addition to the above required encryption ciphers, it is recommended that the $\mathrm{SSH}$ implementation also supports:

- AEAD_AES_256_GCM (As per Suite B support in rfc6239)

- AEAD_AES_128_GCM (As per Suite B support in rfc6239)

\subsubsection{HMACS}

The SSH implementation shall support the following HMAC data integrity algorithms (only when not using an AEAD mode cipher which is the preferred method to meet NISTIR 7628 Cryptographic requirements for the Smart Grid:

- HMAC-SHA1 (required by RFC)

The MD5 cryptographic hash function is considered cryptographically broken and shall not be used for calculation of the message authentication code.

\subsubsection{Key Exchange Methods}

The SSH implementation shall support at least one of the following Key Exchange Methods:

- diffie-hellman-group14-sha1 (required by RFC)

- diffie-hellman-group1-sha1 (required by RFC)

In addition to the above required Key Exchange Methods, it is recommended that the SSH implementation also supports:

- $\quad$ ecdh-sha2-nistp384 (As per Suite B support in rfc6239)

- ecdh-sha2-nistp256 (As per Suite B support in rfc6239)

\subsubsection{Server Hostkeys}

The SSH implementation shall support the following Server Hostkey algorithms and shall advertise them in the following order of preference:

- $\quad$ x509v3-ecdsa-sha2-nistp384

- $\quad$ x509v3-ecdsa-sha2-nistp256 (As per Suite B support in rfc6239)

- $\quad$ ssh-rsa (note: It is recommended to use at least a key size of 3072bit for 128bit level security)

- $\quad$ ssh-dss (note: It is recommended to use at least a key size of 3072bit for 128bit level security) (required by RFC)

\footnotetext{
${ }^{3}$ http://csrc.nist.gov/publications/nistpubs/800-131A/sp800-131A.pdf
} 


\subsubsection{Compression Algorithms}

The SSH implementation shall support the following compression algorithms and shall advertise them in the following order of preference:

- None (required by RFC)

- zlib

\subsubsection{Authentication Methods}

The SSH implementation shall support the following authentication methods:

- Password

- Publickey (Required by RFC)

\subsubsection{Publickey Method}

The SSH implementation shall always fail to authenticate users attempting to authenticate via the publickey method.

\subsubsection{Password Method}

The SSH implementation shall allow at most 3 attempts to authenticate via the password method. If the SSH implementation is acting as a "Server", incoming user authentication information shall be validated against the devices configured user base. If the SSH implementation is acting as a "Client", it will provide username and password information to the remote server as provided by the configuration.

\subsubsection{Connection Channel Methods}

The SSH implementation shall support the following connection service channel methods:

- session

\subsubsection{Session Method}

The SSH implementation shall only support "shell" type session methods, however, no terminal processing shall be done on the incoming data. All other session types shall be rejected. Extended data shall be treated as normal data in the session.

\subsection{Processing requirements}

\subsubsection{Key Renegotiation}

Cipher and data integrity keys shall be renegotiated as recommended by the standard to protect against key reverse engineering. The keys shall be renegotiated if one of the following conditions is met since the last negotiation:

- approximately $2^{\wedge} 31$ packets have been sent,

- approximately $2^{\wedge} 31$ packets have been received,

- approximately 24 hours have elapsed.

\subsubsection{Ignore Packet Stuffing}

SSH_MSG_IGNORE packets shall be inserted into the data stream, with random payload data, if more than $\overline{5}$ minutes have elapsed since the last packet has been sent. The IGNORE packet shall be sent immediately before the next valid packet.

\subsubsection{Channel Window Size}

All channels shall support a receive maximum window size of 4096 bytes, and shall support a maximum packet size of 512 bytes. 


\subsubsection{Process Authorization (systems with an OS)}

The SSH server shall not run at a level of privilege giving its binaries direct access to resources not allowed outside of the intended process.

The SSH server shall not authenticate attempted access by credentials with sufficient privilege to violate or redefine allowed process boundaries.

\subsubsection{Connection keepalive}

The SSH server shall be configured to not use TCP keepalive.

\subsubsection{Connection rate}

The SSH server shall be capable of governing maximum authentication attempt rate.

The SSH server shall be capable of limiting the maximum number of simultaneous connections.

\subsubsection{Prime Numbers / RNG}

Credentials and keys for use with SSH shall be generated from a system with sufficient entropy.

\subsubsection{Private Key Encryption}

Private Keys stored on client systems shall be encrypted with one of (in order of preference): AEAD_AES_256_GCM, AES_256_CBC, AEAD_AES_128_GCM, AES_128_CBC if AES-256 is not available.

Private Keys to be used by human-driven credentials shall not be stored on the client system leveraging them for access.

\section{References:}

The SSH protocol is defined by the Network Working Group of the IETF in several RFCs:

- RFC4250 - The Secure Shell (SSH) Protocol Assigned Numbers

- RFC4251 - The Secure Shell (SSH) Protocol Architecture

- $\quad$ RFC4252 - The Secure Shell (SSH) Authentication Protocol

- RFC4253 - The Secure Shell (SSH) Transport Layer Protocol

- RFC4254 - The Secure Shell (SSH) Connection Protocol

- RFC6239 - Suite B Crypto Suites for SSH 



\section{APPENDIX C: INTEROPERABLE CONFIGURATION PROFILE FOR LDAP}

Following is the contents of the most recent Interoperable Configuration Profile for LDAP as of the date of this publication.

\section{Definition}

Authentication is the process of verifying the digital identity of a user. Central authentication is the process of a Central Authentication Server (CAS) or servers holding all the digital identity credentials for all authorized system users and their corresponding authorization. Every client in the system asks this central authority to verify the digital identity of any new incoming communication attempts.

\section{Authentication}

This document describes a standardized implementation of central authentication suitable for use within control systems utilized within the electric sector. This document applies to all routable communication paths for engineering access. For example, if an engineer wants to access a relay across an IP telnet connection the relay will pass the user name and password the engineer sends to it to the central server to verify they are authorized to have access. Depending on the server's response, the relay will allow or block access to the engineer.

Authentication is essential for security in control systems and to comply with regulatory standards. Authentication allows the control system to verify the identity and authorization of a person before granting access. Authentication also allows the system to create a detailed audit list of who made what changes or executed which commands and when.

\section{Authentication Implementation Overview}

Central authentication enables control system owners to effectively manage and update account information in a timely manner. Central authentication allows administrators to change accounts at one central location and affect the access capabilities of every IED on that system. This is very common and considered best practice for corporate information systems.

\section{Central Authentication}

Shall use lightweight directory access protocol version 3 (LDAPV3) or higher version that allows backward compatibility to version 3 . The technical details of LDAPV3 are documented in a request for comment (RFC) by the internet engineering task force (IETF). The LDAP RFCs were developed to ensure interoperability and consistent designs. The root RFC is RFC4510 and is available online at $<\mathrm{http}: / /$ tools.ietf.org/html/rfc4510>. The technical details for authentication can be found in RFC4513 and is available online at http://tools.ietf.org/html/rfc4513.

The minimum authentication method that shall be used is "Name/Password authentication mechanism of simple bind" found in section 5.1.3 of RFC4513

\section{Additional Security Implementation Requirements}

\subsection{TLS Requirement}

Shall use transport layer security (TLS) when establishing a connection to the central server. This is outlined in RFC4513. LDAP does not have any built-in confidentiality technology so TLS shall be used to encrypt the traffic between the client and server to ensure confidentiality and authenticate the connection to the server. 


\subsection{Client and Server Side Certificate}

Only server side certificates shall be required. Clients will authenticate by the user entering a user name and password which eliminates the need for clients to authenticate as part of the TLS protocol.

\subsection{Anonymous Authentication}

Anonymous Authentication is part of the LDAP server policy and may be preferable to some customers instead of providing a LDAP service account. The LDAP PAM module shall support both authenticated and anonymous authentication methods for the purpose of finding the user's Distinguished Name (DN).

\subsection{Unauthenticated Authentication}

Do not allow "unauthenticated authentication" as per section 5.1.2 in RFC4513. This will prevent any authentication with a proper user name but with a blank password.

\subsection{Custom Database Schema}

Shall not require a custom schema for use with authentication in the CAS database.

\subsection{CAS Stored Data}

Information that shall be required to store in the CAS database is the user name, password, and groups the user is a member of.

\subsection{Mapping of CAS Groups to Product Setting Access}

There shall be settings to map the groups in the CAS to the local groups.

\subsection{Static Database Search Parameters}

There shall be settings in products to set the static database search paths.

The device shall perform an anonymous binding to the LDAP server to locate the LDAP record for Joe. Once the LDAP record is identified, the device will attempt to re-bind with the user's DN and the usersupplied password. If the re-bind is successful, the user has been successfully authenticated. If the rebind fails, the user is denied access.

\subsection{CAS Unavailability}

When the CAS is unavailable the end point device shall use local accounts only for logins authentication.

\subsection{No Automatic Local Account Updates}

When the CAS returns data for an account that matches an account stored locally the device shall not update the local account with the data received from the CAS.

\subsection{Required TLS Algorithms}

TLS shall support AES (128 or 256) and SHA-1/256 or MD5. 


\section{Flow Diagram}

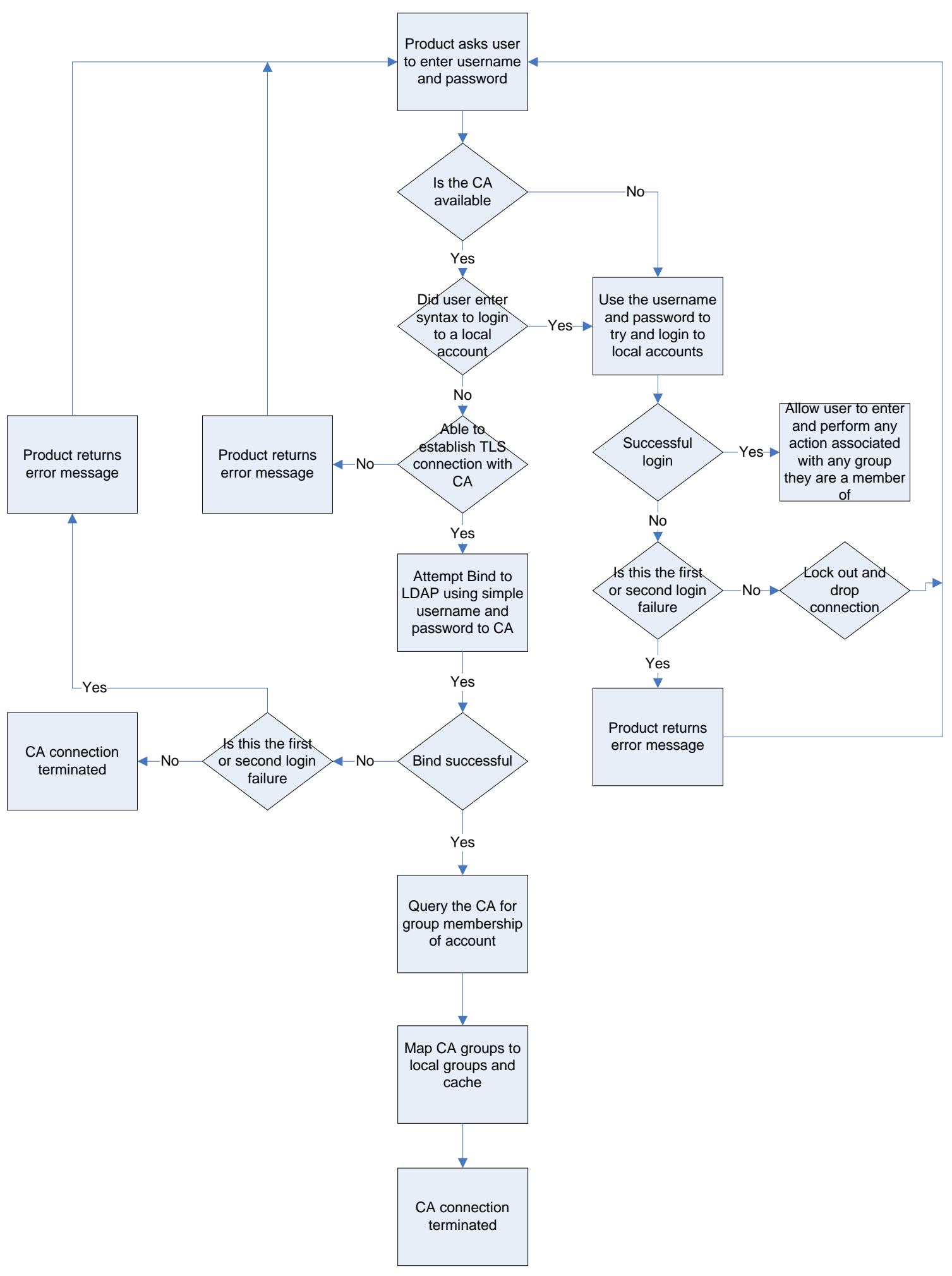

Figure C-3

LDAP Flow Diagram 


\section{Libraries}

\subsection{LDAPv3 Library:}

LDAP is an open protocol that is well documented. All the major players like Sun, Microsoft, Fedora, Netscape, and Novell support LDAPv3. LDAPv3 can interface with Active Directory environments allowing the knowledge base that our customers already have in their IT departments to be used to install central authentication for their control systems.

\subsection{TLS Library}

TLS is an open protocol that is well documented. The Internet engineering task force has documented TLS in RFC 4346. 


\section{APPENDIX D: STANDARDIZATION OF SYSLOG MESSAGE}

\section{Background}

The initial effort of the Lemnos project identified Syslog, IETF RFC 3164, as the standard mechanism for exchanging log messages. The scope of this initial effort only covered the use of Syslog and did not prescribe any specific configuration parameters therefore no formal ICP document was developed.

During this time, feedback from one stakeholder in the oil and gas industry identified specific challenges encountered by the end users when attempting to correlate log messages from multiple devices at multiple geographic locations such as:

- Lack of time zone clarity in message

- Lack of consistency in message content

These items were validated by several stakeholders in the electric utility industry and during the third year of Lemnos, work as described in this summary was done by the Lemnos team, aided by input and feedback from the UCA SG Security Cybersec- Interop Task Force that was formed as an industry outreach arm of Lemnos.

\section{Lemnos Research and Development Efforts}

1. Upon first glance, it was conceivable that if industry adopted an updated Syslog RFC, IETF RFC 5424, which was finalized in 2009 and intended to supersede RFC 3164, most of the problems related to Syslog would be solved. RFC 5424 has a UTP date/timestamp that would indicate timezone, etc. RFC 5424 also has structured data in its first comments field, in the form of (Parameter $=$ Value $)$, where, for instance, a log message for failed login could be created and standardized. A proposal for this effort was written and reviewed with the Lemnos Team and vendors.

2. During Year Three of Lemnos, a UCA SG Security Cybersec-Interop Task Force was proposed and chartered to be the industry outreach arm of Lemnos. During a webinar with the Task Force, and incorporating the previous input of the Lemnos core team and participating vendors, it was decided that the vendors thought RFC 5424 was "ahead of its time", and vendors wanted the Lemnos team to stick with RFC 3164 and improve it. This was a key decision for the project.

3. Work was redirected back to standardizing and improving RFC 3164. The Lemnos core team, with strong input from Schweitzer Engineeing Laboratories, generated a list of 37 proposed standard text messages that would serve as the "first round" of Lemnos standardizations, to be adopted by industry. In a sense, this list was a trial "Interoperable Configuration Profile (ICP)" for Syslog standardization. Also in this list of proposals, the applicable NERC-CIP standards were also included, to help the industry use the Lemnos Syslog work to help with NERC compliance. These messages are shown in Table D-1.

\begin{tabular}{|c|c|c|l|}
\hline $\begin{array}{c}\text { Event } \\
\text { Type }\end{array}$ & Tag Name & NERC CIP & \multicolumn{1}{c|}{ Message } \\
\hline & Event relational tag & & Message in the description of the log \\
\hline & & CIP007 R6 & $\begin{array}{l}\text { Settings for Time Zone were modified by } \\
\text { <username }>\text { at }<\text { remote_ip }>\end{array}$ \\
\hline
\end{tabular}




\begin{tabular}{|c|c|c|c|}
\hline $\begin{array}{l}\text { Event } \\
\text { Type }\end{array}$ & Tag Name & NERC CIP & Message \\
\hline & Event relational tag & & Message in the description of the log \\
\hline Date_Time & DateTimeConfig & $\begin{array}{l}\text { CIP007 R6 } \\
\text { CIP003 R6 }\end{array}$ & $\begin{array}{l}\text { Date/Time of the System set manually by } \\
<\text { username }>\text { at <remote_ip }>\end{array}$ \\
\hline Power & Power & $\begin{array}{l}\text { CIP007 R6 } \\
\text { CIP003 R6 }\end{array}$ & $\begin{array}{l}\text { Device rebooted by < username }>\text { at } \\
<\text { remote_ip> }\end{array}$ \\
\hline Firewall & Firewall & $\begin{array}{l}\text { CIP007 R6 } \\
\text { CIP003 R6 } \\
\text { CIP005 R2 }\end{array}$ & $\begin{array}{l}\text { Firewall general rules were modified by } \\
<\text { username }>\text { at <remote_ip }>\end{array}$ \\
\hline Firewall & Firewall & $\begin{array}{l}\text { CIP007 R6 } \\
\text { CIP003 R6 } \\
\text { CIP005 R2 }\end{array}$ & $\begin{array}{l}\text { Firewall rule <alias }>\text { added by < username }> \\
\text { at <remote_ip }>\end{array}$ \\
\hline Firewall & Firewall & $\begin{array}{l}\text { CIP007 R6 } \\
\text { CIP003 R6 } \\
\text { CIP005 R2 }\end{array}$ & $\begin{array}{l}\text { Firewall rule < alias }>\text { removed by } \\
<\text { username }>\text { at }<\text { remote_ip }>\end{array}$ \\
\hline Firewall & Firewall & $\begin{array}{l}\text { CIP007 R6 } \\
\text { CIP003 R6 } \\
\text { CIP005 R2 } \\
\end{array}$ & $\begin{array}{l}\text { Firewall rule <alias }>\text { modified by } \\
<\text { username }>\text { at }<\text { remote_ip }>\end{array}$ \\
\hline Firmware & Update & $\begin{array}{l}\text { CIP007 R6 } \\
\text { CIP003 R6 }\end{array}$ & $\begin{array}{l}\text { Firmware update initiated by <username }> \\
\text { at <remote_ip> }\end{array}$ \\
\hline VPN & IPsecMgt & $\begin{array}{l}\text { CIP005 R1 } \\
\text { CIP011 R19 }\end{array}$ & $\begin{array}{l}\text { IPsec connection <local_gateway > - } \\
<\text { remote_gateway> generated by } \\
\text { < username }>\text { at }<\text { remote_ip }>\end{array}$ \\
\hline VPN & IPsecMgt & $\begin{array}{l}\text { CIP007 R6 } \\
\text { CIP003 R6 }\end{array}$ & $\begin{array}{l}\text { IPsec connection <local-gateway }>\text { - } \\
<\text { remote-gateway }>\text { modified by } \\
<\text { username }>\text { at }<\text { remote_ip }>\end{array}$ \\
\hline VPN & IPsecMgt & CIP011 R19 & $\begin{array}{l}\text { IPsec connection <local-gateway }>\text { - } \\
<\text { remote-gateway }>\text { removed by } \\
<\text { username }>\text { at }<\text { remote_ip }>\end{array}$ \\
\hline LDAP & LDAPConfig & $\begin{array}{l}\text { CIP007 R6 } \\
\text { CIP003 R6 }\end{array}$ & $\begin{array}{l}\text { Settings created by <username }>\text { at } \\
<\text { remote_ip }>\end{array}$ \\
\hline LDAP & LDAPConfig & $\begin{array}{l}\text { CIP007 R6 } \\
\text { CIP003 R6 }\end{array}$ & $\begin{array}{l}\text { LDAP settings modified by < username }>\text { at } \\
<\text { remote_ip }>\end{array}$ \\
\hline LDAP & LDAPConfig & $\begin{array}{l}\text { CIP007 R6 } \\
\text { CIP003 R6 }\end{array}$ & $\begin{array}{l}\text { BindDN password changed by <username }> \\
\text { at <remote_ip }>\end{array}$ \\
\hline LDAP & LDAPConfig & $\begin{array}{l}\text { CIP007 R6 } \\
\text { CIP003 R6 }\end{array}$ & $\begin{array}{l}\text { LDAP server < hostname }>\text { added by } \\
<\text { username }>\text { at }<\text { remote_ip }>\end{array}$ \\
\hline LDAP & LDAPConfig & $\begin{array}{l}\text { CIP007 R6 } \\
\text { CIP003 R6 }\end{array}$ & $\begin{array}{l}\text { LDAP group mapping modified by } \\
<\text { username }>\text { at }<\text { remote_ip }>\end{array}$ \\
\hline
\end{tabular}




\begin{tabular}{|c|c|c|c|}
\hline $\begin{array}{l}\text { Event } \\
\text { Type }\end{array}$ & Tag Name & NERC CIP & Message \\
\hline & Event relational tag & & Message in the description of the log \\
\hline $\begin{array}{l}\text { User } \\
\text { Accounts }\end{array}$ & UserManagement & $\begin{array}{c}\text { CIP007 R6 } \\
\text { CIP003 R6 } \\
\text { CIP011 R8 } \\
\text { R7 R10 } \\
\end{array}$ & $\begin{array}{l}\text { Created User: <created_username > by } \\
\text { <username }>\text { at }<\text { remote_ip }>\end{array}$ \\
\hline $\begin{array}{l}\text { User } \\
\text { Accounts }\end{array}$ & UserManagement & $\begin{array}{c}\text { CIP007 R6 } \\
\text { CIP003 R6 } \\
\text { CIP011 R8 } \\
\text { R7 R10 }\end{array}$ & $\begin{array}{l}\text { Deleted User: <deleted_username }>\text { by } \\
<\text { username }>\text { at }<\text { remote_ip }>\end{array}$ \\
\hline $\begin{array}{l}\text { User } \\
\text { Accounts }\end{array}$ & UserManagement & $\begin{array}{c}\text { CIP007 R6 } \\
\text { CIP003 R6 } \\
\text { CIP011 R8 } \\
\text { R7 R10 } \\
\end{array}$ & $\begin{array}{l}\text { Modified User: <modified_username }>\text { by } \\
<\text { username }>\text { at }<\text { remote_ip }>\end{array}$ \\
\hline $\begin{array}{l}\text { User } \\
\text { Accounts }\end{array}$ & Login & $\begin{array}{c}\text { CIP007 R5, } \\
\text { R6 CIP011 } \\
\text { R18 }\end{array}$ & $\begin{array}{l}\text { Login successful by: <username }>\text { at } \\
<\text { remote_ip }>\end{array}$ \\
\hline $\begin{array}{l}\text { User } \\
\text { Accounts }\end{array}$ & Login & $\begin{array}{c}\text { CIP007 R5, } \\
\text { R6 CIP011 } \\
\text { R18 } \\
\end{array}$ & Invalid login attempt from <remote_ip $>$ \\
\hline Network & NetworkConfig & $\begin{array}{l}\text { CIP007 R6 } \\
\text { CIP003 R6 } \\
\text { CIP005 R2 }\end{array}$ & $\begin{array}{l}\text { Global network settings modified by: } \\
\text { <username }>\text { at }<\text { remote_ip }>\end{array}$ \\
\hline Network & NetworkConfig & $\begin{array}{l}\text { CIP007 R6 } \\
\text { CIP003 R6 } \\
\text { CIP005 R2 }\end{array}$ & $\begin{array}{l}\text { Network interface <alias }>\text { modified by } \\
<\text { username }>\text { at }<\text { remote_ip }>\end{array}$ \\
\hline Network & NetworkConfig & $\begin{array}{l}\text { CIP007 R6 } \\
\text { CIP003 R6 } \\
\text { CIP005 R2 }\end{array}$ & $\begin{array}{l}\text { Network address <alias }>\text { created by } \\
<\text { username }>\text { at }<\text { remote_ip }>\end{array}$ \\
\hline Network & NetworkConfig & $\begin{array}{l}\text { CIP007 R6 } \\
\text { CIP003 R6 } \\
\text { CIP005 R2 }\end{array}$ & $\begin{array}{l}\text { Network Address < <alias }>\text { modified by } \\
<\text { username }>\text { at }<\text { remote_ip }>\end{array}$ \\
\hline Network & NetworkConfig & $\begin{array}{l}\text { CIP007 R6 } \\
\text { CIP003 R6 }\end{array}$ & $\begin{array}{l}\text { Network address < alias }>\text { deleted by } \\
<\text { username }>\text { at < remote_ip }>\end{array}$ \\
\hline Syslog & SyslogConfig & $\begin{array}{l}\text { CIP005 R3 } \\
\text { CIP007 R6 } \\
\text { CIP003 R6 } \\
\end{array}$ & $\begin{array}{l}\text { Settings for Syslog modified by } \\
<\text { username }>\text { at <remote_ip > }\end{array}$ \\
\hline Syslog & SyslogConfig & $\begin{array}{l}\text { CIP005 R3 } \\
\text { CIP007 R6 } \\
\text { CIP003 R6 }\end{array}$ & $\begin{array}{l}\text { Syslog destination < alias }>\text { created by } \\
<\text { username }>\text { at }<\text { remote ip }>\end{array}$ \\
\hline
\end{tabular}




\begin{tabular}{|c|c|c|c|}
\hline $\begin{array}{l}\text { Event } \\
\text { Type }\end{array}$ & Tag Name & NERC CIP & Message \\
\hline & Event relational tag & & Message in the description of the log \\
\hline Syslog & SyslogConfig & $\begin{array}{l}\text { CIP005 R3 } \\
\text { CIP007 R6 } \\
\text { CIP003 R6 }\end{array}$ & $\begin{array}{l}\text { Settings for Syslog destination <alias }> \\
\text { modified by < username }>\text { at }<\text { remote_ip }>\end{array}$ \\
\hline Syslog & SyslogConfig & $\begin{array}{l}\text { CIP005 R3.2 } \\
\text { CIP007 } \\
\text { R5.1.2 } \\
\text { CIP011 R18 } \\
\end{array}$ & $\begin{array}{l}\text { Syslog destination <alias > deleted by } \\
<\text { username }>\text { at <remote_ip }>\end{array}$ \\
\hline Certificate & X.509CertMgt & $\begin{array}{l}\text { CIP005 R1, } \\
\text { R2 CIP007 } \\
\text { R6 }\end{array}$ & $\begin{array}{l}\text { X.509 Certificate/Private key <alias }> \\
\text { generated by < username }>\text { at }<\text { remote_ip }>\end{array}$ \\
\hline Certificate & X.509CertMgt & $\begin{array}{l}\text { CIP005 R1, } \\
\text { R2 CIP007 } \\
\text { R6 }\end{array}$ & $\begin{array}{l}\text { X.509 Certificate/Private key <alias> } \\
\text { uploaded by < username> at < remote_ip> }\end{array}$ \\
\hline Certificate & X.509CertMgt & $\begin{array}{l}\text { CIP005 R1, } \\
\text { R2 CIP007 } \\
\text { R6 }\end{array}$ & $\begin{array}{l}\text { X.509 Certificate <alias > downloaded by } \\
<\text { username }>\text { at }<\text { remote_ip }>\end{array}$ \\
\hline Certificate & X.509CertMgt & $\begin{array}{l}\text { CIP005 R1, } \\
\text { R2 CIP007 } \\
\text { R6 }\end{array}$ & $\begin{array}{l}\text { X.509 Certificate/Private key <alias> } \\
\text { deleted by < username> at <remote_ip> }\end{array}$ \\
\hline Certificate & X.509CertMgt & $\begin{array}{l}\text { CIP005 R1, } \\
\text { R2 CIP007 } \\
\text { R6 }\end{array}$ & $\begin{array}{l}\text { X.509 Certificate/Private key <alias> has } \\
\text { expired; IPsec communications may be } \\
\text { affected }\end{array}$ \\
\hline LDAP & LDAPConfig & CIP011 R14 & $\begin{array}{l}\text { LDAP failed connection to }<\text { hostname }>\text { at } \\
<\text { Remote IP }>\end{array}$ \\
\hline $\begin{array}{l}\text { User } \\
\text { Accounts }\end{array}$ & UserManagement & CIP011 R10 & $\begin{array}{l}\text { Password changed }<\text { Username }>\text { at } \\
<\text { Remote_IP }>\end{array}$ \\
\hline
\end{tabular}

Table D-1

Proposed Syslog Standardized Messages

4. A comparison chart of the 37 proposed messages completed by two Lemnos participating vendors, Garrettcom and Phoenix Contact, showed the wide disparity of just two of the vendor messages with the Lemnos standard messages. It was obvious that if we continued the process and eventually had all nine of the vendors fill out a comparison chart, very little if anything would match or correlate. We were told by the team that the process of changing syslog message text by a vendor would involve a lengthy process of re-writing code for all the messages; it was not a simple process where, for instance, new text might be inserted in a software matrix in the program.

5. It was judged by the Lemnos team to be impractical, and require an enormous amount of work, to try and have all Lemnos participating vendors complete Syslog changes within the one year remaining on the project. The situation with Syslog was very different than with Internet communication protocols IPsec or SSH, where vendors only had to adopt Lemnos ICP settings with their existing equipment, and did not have to go in and change code (except in the few cases where cryptographic algorithms had to be changed). 
6. An alternate way to provide standardized Syslog messaging for industry became apparent at the EPRI Lemnos interoperability demo in Knoxville, TN in August. Vendor Alien Vault offered an open source SIEM (Security Information and Event Manager) called OSSIM, which had the capability of taking in log messages from many different Lemnos participating vendors, including Cisco, Ruggedcom, etc. and outputting uniform and correlated messages in plain English in a chart-like display. The free, open source product may be viewed at http://alienvault.com/products/open-source-siem . 



\section{APPENDIX E: SECURITY CORE FUNCTION AND DEFINITION}

\section{Security core functional and nonfunctional Requirements}

Functional requirements describe what are being performed. This is a set of binary questions, as the system either does something or it does not. There is usually no measurement or metric involved. Nonfunctional requirements assign metrics and measurements to the functions. They describe characteristics like how well, how much, and how fast specific functions are performed. The nonfunctionals map to functional requirements. In Appendix A, A1 is a list of functional and the corresponding nonfunctional requirement. Not all functional requirements have a mapping to a nonfunctional.

\section{Functional/Nonfunctional Mapping}

The Lemnos security architecture building block is the core security function. The core security functions that will be implemented in the reference architecture and included in the vendor implementation are:

\begin{tabular}{|l|l|l|}
\hline CORE SECURITY FUNCTION & HOW IMPLEMENTED & REQUIREMENTS \\
\hline $\begin{array}{l}\text { Secure Communications } \\
\text { Channel }\end{array}$ & VPN with IPSEC Protocol & $2,3,4,5,6,7,26,41,43$, \\
\hline Messaging & SYSLOG & 9,37 \\
\hline
\end{tabular}

The following core security functions have been envisioned but will not be implemented, and are not described in depth:

\begin{tabular}{|l|l|}
\hline CORE SECURITY FUNCTION & REQUIREMENTS \\
\hline Network Intrusion Detection (NIDS) & 27,31 \\
\hline Host Intrusion Detection (HIDS) & 28 \\
\hline Antivirus & 47 \\
\hline
\end{tabular}

There are many other requirements in the master table that are not describing core security functions but are necessary for equipment operability and security.

\begin{tabular}{|l|l|}
\hline SECURITY OR OPERATIONAL FEATURE & REQUIREMENT \\
\hline Startup testing & 13,14 \\
\hline Storage through Power Cycle & 10,12 \\
\hline Date and Time & $24,32,33$ \\
\hline Diagnosis & 1,13 \\
\hline Security & $15,16,17,18,19,20,21,22,43,48$ \\
\hline Configuration & $11,30,48$ \\
\hline
\end{tabular}

\section{Interoperability Definition}

Outside vendors equipment wishing to interoperate with Lemnos compliant equipment should have the following functionality:

\begin{tabular}{|l|l|l|}
\hline CORE SECURITY FUNCTION & HOW IMPLEMENTED & REQUIREMENTS \\
\hline $\begin{array}{l}\text { Secure Communications } \\
\text { Channel }\end{array}$ & VPN with IPSEC Protocol & $2,3,4,5,6,7$ \\
\hline Messaging & SYSLOG & 9,37 \\
\hline
\end{tabular}




\section{Definitions and Terms}

The definitions and terms are collections of technologies listed during the functional requirements list phase.

1. Access Control List (ACL)-users, groups, machines and process that have been given access to a resource(NIST IR 7298)

2. AES-Advanced Encryption Standard-A symmetric block cipher for encrypting and decrypting information.(NIST 1R 7298)

3. Alert Generation-the process of a device sending or generating status and alert messages, either to a central Syslog server or storing the messages locally.

4. Asset-tangible or intangible entity that has value to an organization(Catalog of Control Systems Security)

5. Authentication-confirming the identity of a user, process, or a device before access to resources is granted. (NIST IR 7298, 800-53)

6. Authenticity-confidence and trust that and entity has been verified and is genuine (NIST SP80082)

7. Authorization-rights and permissions granted to an entity, process, or system to access a control (Catalog of Control Systems Security)

8. Availability-resource providing timely and reliable access to information(NIST SP800-82)

9. Certificate Authority-entity that issues and revokes public key certificates(NIST IR 7298)

10. Confidentiality-assurance that only authorized individuals, process, and devices have accessed information (Catalog of Control System Security)

11. Cryptography-the principles, means and methods for transforming data to hide and prevent modification from unauthorized users(NIST SP800-82)

12. Denial of Service(DoS)-Prevention or disruption of authorized access to a system, which delays system operations or process function(ISA99)

13. DHCP-Dynamic Host Configuration Protocol-protocol used to assign IP address to nodes(NIST SP800-82)

14. DMZ-Demilitarized Zone-External facing network and systems with interfaces located in un-trusted networks

15. Encryption-conversion of data into ciphertext, for security or privacy, which cannot be understood by unauthorized entities. (NIST SP800-82)

16. ESP-Electronic Security Perimeter-Logical border surrounding a network for which access in controlled (Catalog of Control Systems Security)

17. Event Storage-centralized storage and collection of system generated event messages 
18. Event-an observable occurrence in an information system, usually monitored and generates an alarm to an event logging system (NIST SP800-82)

19. Firewall- a gateway that limits traffic between networks in accordance with security policies. Can be software or hardware. (NIST SP800-82)

20. HMI-Human Machine Interface-Hardware and/or software through which one interacts with a system or controller(NIST SP 800-82)

21. Identification-the process of verifying the identity of a device, user, or process before granting access to a resource. (NIST SP800-82)

22. Identity-a unique name that identifies and individual or service, with sufficient information to make the name unique (NIST SP800-82)

23. IED-Intelligent Electronic Device-A device capable of receiving or sending data or control information (NIST SP 500-82)

24. Interoperability-The ability of two or more systems or components to exchange information and to use the information that has been exchanged

25. IPSec-IP Security-the framework for securing IP traffic, including key management, for protection of Virtual Private Network communications, including the type of security for the VPN (NIST IR 7298)

26. Local Area Network (LAN)-communications infrastructure designed to connect computers and other communication devices, limited to a specific geographical location(ISA99)

27. Metric-Measurement used to quantify a component (www.thefreedictionary.com/metrics)

28. NIDS-Network Intrusion Detection System-software that looks for certain suspicious communication activity, based on predefined malicious signatures (NIST IR 7298)

29. OCSP-Online Certificate Status Protocol-Protocol used in the revocation status of X.509 digital certificates.( RFC 2560)

30. OPSAID-Open PCS Security Architecture for Interoperable Design-Joint government/industry project to develop a security architecture utilizing open source software and hardware. (http://www.automationworld.com/view-2974)

31. Password-a sting of characters used to authenticate identity or verify authorization(Catalog of Control Systems Security)

32. Proxy/Gateway-application that breaks a direct connection between server and client. This provides an indirect path from external to internal networks; acts as the entrance point to another network. (Catalog of Control System Security) (NIST SP800-82)

33. Remote Access-external access by users or processes outside the electronic security perimeter of the network(Catalog of Control Systems Security) 
34. Router-a device that connects to physically different networks. Usually used to connect wide area networks.

35. SCADA-Supervisory Control and Data Acquisition-hardware and software used to acquire data for the purpose of monitoring and control (Catalog of Control Systems)

36. SNMP-Simple Network Management Protocol-protocol used for managing network devices; including monitoring network performance, packet loss, and error rates (NIST SP800-82)

37. SSL-Secure Sockets Layer-provides a secure encrypted channel between two devices(also known as HTTPS)(NIST SP 800-82)

38. Switch-an OSI layer 2/3 device that interconnects devices(Catalog of Control Systems Security)

39. TLS-Transport Layer Security-see Secure Socket Layer (SSL)

40. Use Case-technique for capturing functional and non-functional requirements and conveys how a system should interact with another system or end-user(ISA99)

41. VPN-Virtual Private Network-logical network that is established over an existing physical untrusted network, by virtual tunneling across the real network (NIST IR 7298, NIST SP 800-82))

42. W3C-World Wide Web Consortium-Develops interoperable technologies, including specifications, guidelines, and tools, for the web.(www.w3c.org)

43. X.509 Certificate-public key, that is unforgeable by the digital signature of the certification authority that issued the certificate(NIST IR 7298)

\section{System Test Scenarios}

\section{VPN component test scenarios}

\section{Test Scenario \#1 - Pre Shared Passphrase}

A VPN is configured between two Lemnos devices with the pre shared passphrase $X X X X X X X X$. The Lemnos device on the left is the SNL reference implementation and the OPSAID on the right is the SEL implementation. The two Lemnos devices communicate with each other through a WAN to establish Security Associations (SA) for the VPN tunnel. Once the SAs have been established the 192.168.1.0/24 network can securely communicate with the 10.0.1.0/24, with the encrypted channel represented by the green link between Lemnos devices. At the same time, syslog-ng messages are being sent to SNL Lemnos Master from both Lemnos devices.

\section{Test Scenario \#2 - Pre Shared X.509 Certs}

A VPN is configured between two Lemnos devices, each possessing their own X.509 public / private key pair and the public key of the opposite Lemnos. The Lemnos device on the left is the SNL reference implementation and the Lemnos on the right is the SEL implementation. The two Lemnos devices communicate with each other through a WAN to establish Security Associations (SA) for the VPN tunnel. Once the SAs have been established the 192.168.1.0/24 network can securely communicate with the 10.0.1.0/24, with the encrypted channel represented by the green link between Lemnos devices. At the same time, syslog-ng messages are being sent to SNL Lemnos Master from both Lemnos devices. 


\section{Test Scenario \#3 - CA Signed X.509 Certs}

A VPN is configured between two Lemnos devices, each possessing their own CA signed X.509 public I private key pair and the public key of the SNL Lemnos Master. The SNL Lemnos Master device acts as the CA and digitally signs each OPSAIDs certificate so public keys do not have to be distributed prior to the VPN tunnel being established. The CA signature of each certificate verifies that each certificate is authentic. The Lemnos device on the left is the SNL reference implementation and the Lemnos on the right is the SEL implementation. The two Lemnos devices communicate with each other through a WAN to establish Security Associations (SA) for the VPN tunnel. Once the SAs have been established the 192.168.1.0/24 network can securely communicate with the 10.0.1.0/24, with the encrypted channel represented by the green link between Lemnos devices. At the same time, syslog-ng messages are being sent to SNL Lemnos Master from both Lemnos devices.

\section{Test Scenario \#4 - CA Signed X.509 Certs with OCSP}

A VPN is configured between two Lemnos devices, each possessing their own CA signed X.509 public I private key pair and the public key of the SNL Lemnos Master. The SNL Lemnos Master device acts as the CA and digitally signs each Lemnos certificate so public keys do not have to be distributed prior to the VPN tunnel being established. The CA signature of each certificate verifies that each certificate is authentic. The Lemnos device on the left is the SNL reference implementation and the Lemnos on the right is the SEL implementation. The two Lemnos communicate with each other through a WAN to establish Security Associations (SA) for the VPN tunnel. Once the SAs have been established the 192.168.1.0/24 network can securely communicate with the 10.0.1.0/24, with the encrypted channel represented by the green link between Lemnos. If a certificate is added to the Certificate Revocation List (CRL) of the SNL Lemnos Master device then the new CRL will be sent out to each OPSAID device, represented by the blue dashed line. The certificates appearing on the new CRL will no longer be verified by the CA. At the same time, Syslog-ng messages are being sent to SNL Lemnos Master from both Lemnos devices.

\section{Firewall Test Scenarios}

\section{Test Scenario \#1 - No Firewall}

The Lemnos device on the left is the SNL reference implementation and the Lemnos on the right is the SEL implementation. The two Lemnos communicate with each other through a WAN and act as a gateway for each of the end devices connected to them. All communications between host Eve and host MiscBox should be disallowed. However, without a firewall Eve and MiscBox have established a telnet session between each other, represented as the green link between them.

\section{Test Scenario \#2 - Firewall Port Filter}

The Lemnos device on the left is the SNL reference implementation and the Lemnos on the right is the SEL implementation. The two Lemnos devices communicate with each other through a WAN and act as a gateway for each of the end devices connected to them. All communications between host Eve and host MiscBox should be disallowed. However, with a firewall only filtering telnet traffic between Eve and MiscBox only port 23 (represented by the green link) is blocked, while the two parties can still ssh to each other via port 22, represented by the blue link.

\section{Test Scenario \#3 - Firewall IP Filter}

The Lemnos device on the left is the SNL reference implementation and the Lemnos on the right is the SEL implementation. The two Lemnos devices communicate with each other through a WAN and act as a gateway for each of the end devices connected to them. All communications between host Eve and host MiscBox should be disallowed. Now, with a firewall filtering on IP, telnet traffic, ssh traffic, and all other traffic between the IPs of Eve and MiscBox are blocked by the firewall. 


\section{Functional and Nonfunctional Requirements}

\begin{tabular}{|c|c|c|}
\hline & Functional Security Requirement & $\begin{array}{l}\text { Corresponding Non-functional } \\
\text { Security Requirement }\end{array}$ \\
\hline 1.0 & $\begin{array}{l}\text { System shall provide automated diagnostics and } \\
\text { reporting }\end{array}$ & $\begin{array}{l}\text { The system shall provide automated } \\
\text { diagnostics and reporting a minimum of } 1 \text { time } \\
\text { per month }\end{array}$ \\
\hline 2.0 & $\begin{array}{l}\text { The authentication for the secure SCADA/PCS } \\
\text { tunnel shall comply with the IPSec Standard }\end{array}$ & VPN shall provide zero loss of communication \\
\hline 3.0 & $\begin{array}{l}\text { The cryptographic tunnel shall support AES, } \\
\text { 3DES, SHA-1, SHA-256, and be compliant with } \\
\text { strongSwans implementation }\end{array}$ & $\begin{array}{l}\text { Cryptography shall have a minimum } \\
\text { (AES,3DES) } 128 \text { bit encryption }\end{array}$ \\
\hline 4.0 & $\begin{array}{l}\text { The SCADA/PCS tunnel shall support pre-shared } \\
\text { passphrases as a key }\end{array}$ & $\begin{array}{l}\text { The passphrase shall be a minimum of } 10 \\
\text { characters and a maximum of } 128 \text { characters }\end{array}$ \\
\hline 5.0 & $\begin{array}{l}\text { The SCADA/PCS tunnel shall support pre-shared } \\
\text { x509 certificates as a key }\end{array}$ & $\begin{array}{l}\text { The device shall have a minimum key of } 1024 \text {, } \\
\text { with support for } 4028 \text { and } 4096\end{array}$ \\
\hline 6.0 & $\begin{array}{l}\text { The SCADA/PCS tunnel shall support CA signed } \\
\text { x509 certificates as a key }\end{array}$ & \\
\hline 7.0 & Key revocations shall support OCSP & $\begin{array}{l}\text { The system shall check the CRL interval every } \\
10 \text { minutes }\end{array}$ \\
\hline 8.0 & $\begin{array}{l}\text { The device shall be interoperable with other } \\
\text { certified devices }\end{array}$ & \\
\hline 9.0 & $\begin{array}{l}\text { Local events shall be reported in a format } \\
\text { compatible with Syslog }\end{array}$ & \\
\hline 10.0 & Settings shall be stored in nonvolatile memory & $\begin{array}{l}\text { The system shall be configured without } \\
\text { requiring a device reboot }\end{array}$ \\
\hline 11.0 & $\begin{array}{l}\text { There shall be a human readable way to } \\
\text { configure settings (GUI, CLI, or through a PC) is } \\
\text { up to the customer }\end{array}$ & \\
\hline 12.0 & $\begin{array}{l}\text { Date and time shall be maintained through a } \\
\text { power cycle }\end{array}$ & $\begin{array}{l}\text { The device shall hold the date/time setting for } \\
\text { up to } 10 \text { minutes }\end{array}$ \\
\hline 13.0 & $\begin{array}{l}\text { Equipment self tests shall be performed prior to } \\
\text { enabling the equipment }\end{array}$ & $\begin{array}{l}\text { The system shall report once per month, } \\
\text { through and in the form of Syslog messages }\end{array}$ \\
\hline 14.0 & $\begin{array}{l}\text { All self tests shall be performed prior to enabling } \\
\text { the equipment }\end{array}$ & \\
\hline 15.0 & $\begin{array}{l}\text { The device shall provide user based password } \\
\text { security }\end{array}$ & \\
\hline 16.0 & The device shall support password protection & $\begin{array}{l}\text { The device shall support password protection } \\
\text { from 8-128 characters }\end{array}$ \\
\hline 17.0 & $\begin{array}{l}\text { Password protection shall support all printable } \\
\text { characters }\end{array}$ & $\begin{array}{l}\text { Passwords shall support all printable } \\
\text { characters, from } 7 \text { bit ASCIl set }(0 \times 20-0 \times 70)\end{array}$ \\
\hline 18.0 & Passwords shall not be displayed at anytime & \\
\hline 19.0 & $\begin{array}{l}\text { This device shall trigger an alarm for all } \\
\text { successful attempts to login as a user with write } \\
\text { and/or modify rights }\end{array}$ & $\begin{array}{l}\text { The alarm shall be generated and received } \\
\text { within } 30 \text { seconds of the event from the user } \\
\text { logging in }\end{array}$ \\
\hline 20.0 & Warnings for invalid passwords shall be logged & $\begin{array}{l}\text { The alarm shall be generated and received } \\
\text { within } 30 \text { seconds of the invalid password } \\
\text { event }\end{array}$ \\
\hline 21.0 & $\begin{array}{l}\text { Alarm shall be generated after three failed } \\
\text { attempts to provide a legitimate password for an } \\
\text { access request }\end{array}$ & $\begin{array}{l}\text { The alarm shall be generated after } 3 \text { failed } \\
\text { password attempts }\end{array}$ \\
\hline 22.0 & $\begin{array}{l}\text { The device shall provide the ability to lockout } \\
\text { users }\end{array}$ & \\
\hline
\end{tabular}




\begin{tabular}{|c|c|c|}
\hline 23.0 & $\begin{array}{l}\text { The device shall provide a Syslog feature that } \\
\text { logs successful access entries and failed access } \\
\text { attempts }\end{array}$ & $\begin{array}{l}\text { The alarm shall be generated and received } \\
\text { within } 30 \text { seconds of the failed attempts }\end{array}$ \\
\hline 24.0 & $\begin{array}{l}\text { Date and data shall include day,month, and year } \\
\text { and shall compensate for leap years. }\end{array}$ & \\
\hline 25.0 & $\begin{array}{l}\text { Product shall support a mechanism to filter } \\
\text { undesired traffic }\end{array}$ & $\begin{array}{l}\text { Users shall be able to create, at a minimum, } \\
128 \text { rules }\end{array}$ \\
\hline 26.0 & $\begin{array}{l}\text { VPN MIBs shall provide security status } \\
\text { information }\end{array}$ & \\
\hline 27.0 & $\begin{array}{l}\text { Network Intrusion Detection System(NIDS) shall } \\
\text { be targeted to SCADA protocols and updated as } \\
\text { required }\end{array}$ & \\
\hline 28.0 & $\begin{array}{l}\text { Host Intrusion Detection System(HIDS) shall be } \\
\text { considered for future implementation }\end{array}$ & \\
\hline 29.0 & $\begin{array}{l}\text { Event Storage, alert generation, and visualization } \\
\text { shall be supported on remote systems }\end{array}$ & \\
\hline 30.0 & $\begin{array}{l}\text { Device configuration and system logging shall } \\
\text { have a configuration interface. }\end{array}$ & \\
\hline 31.0 & $\begin{array}{l}\text { Network Intrusion Detection System(NIDS) shall } \\
\text { be supported on the device }\end{array}$ & \\
\hline 32.0 & $\begin{array}{l}\text { The system shall have a support for the selection } \\
\text { of a time zone }\end{array}$ & $\begin{array}{l}\text { The system shall support a minimum of } 12 \\
\text { hour offset }\end{array}$ \\
\hline 33.0 & $\begin{array}{l}\text { The system shall support date/time to be } \\
\text { adjusted manually }\end{array}$ & \\
\hline 34.0 & By default, the system shall drop all packets & \\
\hline 35.0 & $\begin{array}{l}\text { Default setting shall include a rule to allow the } \\
\text { user to login to the management interface }\end{array}$ & \\
\hline 36.0 & $\begin{array}{l}\text { The system shall, at a minimum, allow filtering of } \\
\text { IP traffic by the source and destination IP } \\
\text { address range and the source and destination } \\
\text { port number range of TCP and UDP packets }\end{array}$ & The system shall support ports 1-65534 \\
\hline 37.0 & $\begin{array}{l}\text { Syslog events related to the firewall configuration } \\
\text { shall be generated }\end{array}$ & \\
\hline 38.0 & $\begin{array}{l}\text { All Syslog events related to the modification of } \\
\text { settings shall include the username of the user }\end{array}$ & \\
\hline 39.0 & $\begin{array}{l}\text { Allow the user to define the protocol-level static } \\
\text { routes that control the flow of network traffic } \\
\text { through the system }\end{array}$ & \\
\hline 40.0 & $\begin{array}{l}\text { System will be able to forward Syslog events to } \\
\text { the Syslog server via the network }\end{array}$ & $\begin{array}{l}\text { The system shall support a minimum of } 2 \\
\text { Syslog servers }\end{array}$ \\
\hline 41.0 & $\begin{array}{l}\text { The system shall support public and private keys } \\
\text { (using RSA encryption) }\end{array}$ & \\
\hline 42.0 & $\begin{array}{l}\text { The system shall support role based } \\
\text { authorization. }\end{array}$ & $\begin{array}{l}\text { The system shall have a minimum of } 2 \text { roles, } \\
\text { User and Administrator (Admin) }\end{array}$ \\
\hline 43.0 & $\begin{array}{l}\text { The system shall support third party } \\
\text { authentication, such as pass tokens, smart } \\
\text { cards, etc (Future use and implementation) }\end{array}$ & \\
\hline 44.0 & $\begin{array}{l}\text { The system shall support location based } \\
\text { authentication, such as GPS. (Future use and } \\
\text { implementation) }\end{array}$ & \\
\hline 45.0 & $\begin{array}{l}\text { The system shall support automated software } \\
\text { management support, such as software patches, } \\
\text { OS upgrades, etc. (Future use and } \\
\text { implementation) }\end{array}$ & \\
\hline 46.0 & If a BIOS is supported, settings shall be & \\
\hline
\end{tabular}




\begin{tabular}{|l|l|l|}
\hline 47.0 & $\begin{array}{l}\text { password protected (Future use and } \\
\text { implementation) }\end{array}$ & $\begin{array}{l}\text { Anti-Virus software shall be supported on the } \\
\text { implemented platform (Future use and } \\
\text { implementation) }\end{array}$ \\
\hline 48.0 & $\begin{array}{l}\text { Support for backup and recovery shall be } \\
\text { available for configuration/system files (Future } \\
\text { use and implementation) }\end{array}$ \\
\hline
\end{tabular}




\section{APPENDIX F: SECURITY FUNCTIONALITY SELECTION}

\section{Security Functionality Definition}

To support the Lemnos Interoperable Security Program, Sandia is developing an updated version of the OPSAID (Open PCS (Process Control System) Security Architecture for Interoperable Design) reference implementation. OPSAID is a security architecture that incorporates several aspects of security functionality. The overall OPSAID architecture is divided into several areas of security functionality. Examples of areas of security functionality include virtual private networks (VPN), system logging and firewall services. For each area of security functionality, the basic OPSAID philosophy has been to implement the most commonly used, well-proven, open source solution.

An OPSAID Security Function relates to a specific functional role in security, such as virtual private networks (VPN), system logging or firewall services. The currently defined OPSAID security functions are:

- Virtual Private Networking/Encryption

- Firewall Services

- Network Intrusion Detection System

- Host Intrusion Detection System

- Event Logging

- Event Database Storage, Alert Generation \& Visualization

- End-device Configuration Session Logging

- Authentication

- Device Management

The Lemnos security architecture uses these defined security functions as a starting point and has selected a subset of these functions described in the following section. An OPSAID Component Module is a specific implementation of an OPSAID Security Function. For example, for the OPSAID Security Function "Virtual Private Networking/Encryption", one OPSAID Component Module is IPSec. Each Component Module will contain detailed information about configuration and interoperability in its interoperability guide.

\section{Security Functions Selected}

The core security functions to be implemented in the Lemnos reference architecture and included in the vendor implementation are: 


\begin{tabular}{|c|c|c|c|}
\hline FUNCTION & COMPONENT & MODULE & $\begin{array}{l}\text { IMPLEMEN- } \\
\text { TATION }\end{array}$ \\
\hline $\begin{array}{l}\text { Secure } \\
\text { Communications } \\
\text { Channel }\end{array}$ & $\begin{array}{l}\text { Virtual Private Network } \\
\text { (VPN) }\end{array}$ & IPsec & $\begin{array}{l}\text { Reference } \\
\text { Architecture/ } \\
\text { Vendor }\end{array}$ \\
\hline $\begin{array}{l}\text { Filter Illegal Network } \\
\text { Traffic }\end{array}$ & Packet Filter Firewall & IPtables & Vendor \\
\hline $\begin{array}{l}\text { Notification, Non- } \\
\text { repudiation, } \\
\text { Traceability, Trouble } \\
\text { Shooting }\end{array}$ & Audit Log & Syslog & $\begin{array}{l}\text { Reference } \\
\text { Architecture } \\
\text { /Vendor }\end{array}$ \\
\hline $\begin{array}{l}\text { Cryptography and } \\
\text { Password Management }\end{array}$ & Security Enforcement & $\begin{array}{l}\text { IPsec: Certificate } \\
\text { Authorities (CAs), } \\
\text { X.509 Certificates, } \\
\text { shared keys }\end{array}$ & Vendor \\
\hline $\begin{array}{l}\text { Day, Month, Year and } \\
\text { Time Zone }\end{array}$ & Date and Time & $\begin{array}{l}\text { IRIG-B and } \\
\text { UNIX/LINUX date } \\
\text { (default install) }\end{array}$ & Vendor \\
\hline
\end{tabular}

\section{Vendor Implementation}

The Lemnos Interoperable Security Program includes SEL developing, producing, and delivering a security gateway built on the technology started at Sandia in the OPSAID project. Product characteristics include:

- $\quad$ Creates a VPN tunnel between trusted networks using IPsec.

- Contains a "deny by default" firewall that filters incoming TCP, UDP, and ICMP packets based on a configurable set of rules

- Formats and transports event logs according to the Syslog specification to enable interoperability, reporting, and tracking of security events

- Cryptographically secured communications over untrusted Ethernet and Serial Line Internet Protocol (SLIP) channels

- Events are time-stamped and time-synched through an IRIG-B signal that the unit both receives and distributes.

\section{Selection Criteria}

Selection criteria were defined by mapping requirements to the end-user utilizing TVA's specific problem set, and to the vendor community through a matrix of components and modules with core network security functions. 
The Lemnos team collectively decided upon a specific set of security functions by scoring each security function based on the functional value to the end user and rating overall labor effort to maintain the network security equipment. A low functional value score represents a high value to the end user. The labor rating represents the cost of maintaining a component once it is deployed. The VPN security function represented one of the highest values given TVA's business needs. Although scoring lower, the audit log security function was selected because it is a complimentary function. For example, using the audit logging function is the most efficient method to implement a VPN with another function such as Date/Time. 


\begin{tabular}{|c|c|c|c|c|c|c|c|c|c|c|c|}
\hline Function & $\begin{array}{l}\text { Secure } \\
\text { Communications } \\
\text { Channel }\end{array}$ & $\begin{array}{l}\text { Filter Illegal } \\
\text { Network Traffic }\end{array}$ & $\begin{array}{l}\text { Notification, non- } \\
\text { repudiation, } \\
\text { traceability, } \\
\text { trouble shooting }\end{array}$ & $\begin{array}{l}\text { Detect malicious } \\
\text { activity by } \\
\text { monitoring } \\
\text { network traffic }\end{array}$ & $\begin{array}{l}\text { Monitor and } \\
\text { analyze system } \\
\text { processes }\end{array}$ & $\begin{array}{l}\text { Identify, } \\
\text { neutralize or } \\
\text { eliminate } \\
\text { malicious } \\
\text { software } \\
\end{array}$ & $\begin{array}{l}\text { Cryptography and } \\
\text { Password } \\
\text { Management } \\
\end{array}$ & $\begin{array}{l}\text { Day, month, } \\
\text { year and time } \\
\text { zone }\end{array}$ & $\begin{array}{l}\text { Automated } \\
\text { diagnostics and } \\
\text { reporting }\end{array}$ & Visualization & \begin{tabular}{|l} 
Remote \\
Management
\end{tabular} \\
\hline Component & $\begin{array}{l}\text { Virtual Private } \\
\text { Network (VPN) }\end{array}$ & $\begin{array}{l}\text { Packet filter } \\
\text { firewall }\end{array}$ & Audit Log & \begin{tabular}{|l|} 
Network \\
Intrusion \\
Detection (NID) \\
\end{tabular} & $\begin{array}{l}\text { Host Intrustion } \\
\text { Detection (HID) }\end{array}$ & Anti-Virus & \begin{tabular}{|l} 
Security \\
Enforcement
\end{tabular} & Date and Time & Diagnosis & \begin{tabular}{|l} 
Audit \\
Log/Alarm \\
Display \\
\end{tabular} & $\begin{array}{l}\text { Secure Remote } \\
\text { Access }\end{array}$ \\
\hline \begin{tabular}{|l} 
Modules \\
Vendor
\end{tabular} & \begin{tabular}{|l|} 
IPsec \\
N-Dimension
\end{tabular} & Iptables & $\frac{\text { Syslog }}{\text { N-Dimension }}$ & $\frac{\text { snort }}{N-\text { Dimension }}$ & $\begin{array}{l}\text { Non-open source } \\
\text { (vendor specific): } \\
\text { hashcode based on } \\
\text { SHA-1 or MD5: } \\
\text { Open source: } \\
\text { Tripwire, but } \\
\text { carries a large } \\
\text { memory overhead }\end{array}$ & $\begin{array}{l}\text { Unix/Linux } \\
\text { ClamAV } \\
\end{array}$ & $\begin{array}{l}\text { Ipsec: Certificate } \\
\text { Authorities (CAs), } \\
\text { X.509 Certificates, } \\
\text { shared keys }\end{array}$ & \begin{tabular}{|l|} 
\\
Network Time \\
Protocol (NTP) \\
and UNIX/LINUS \\
date (default \\
install) \\
\end{tabular} & \begin{tabular}{|l|} 
Port scan \\
relative to \\
external devices; \\
UNIX/LINUX \\
cron scheduling \\
for internal \\
connection \\
status via Ipsec \\
\end{tabular} & php-Syslog & ssh \\
\hline $\begin{array}{l}\text { Note: Vendor } \\
\text { information was } \\
\text { determined by } \\
\text { examination of } \\
\text { their websites } \\
\text { and brochures } \\
\end{array}$ & $\begin{array}{l}\text { Industrial } \\
\text { Defender }\end{array}$ & & $\begin{array}{l}\text { Industrial } \\
\text { Defender }\end{array}$ & \begin{tabular}{|l} 
Industrial \\
Defender
\end{tabular} & Industrial Defender & 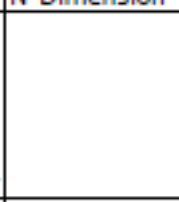 & 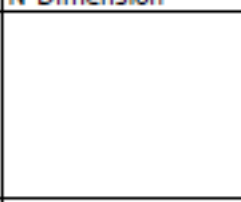 & \begin{tabular}{|l} 
Industrial \\
Defender
\end{tabular} & $\begin{array}{l}\text { Industrial } \\
\text { Defender }\end{array}$ & & \\
\hline & Aegis Odyssey & & Aegis Odyssey & Aegis Odyssey & Aegis Odyssey & & & Aegis Odyssey & Aegis Odyssey & & Aegis Odyssey \\
\hline & Tofino & & Tofino & Tofino & & & & & & & \\
\hline & SEL & SEL & SEL & & & & SEL & SEL & & & SEL \\
\hline & & & & & & & & & & \begin{tabular}{|l|} 
Note: \\
Additional \\
Function is \\
different \\
than a CS \\
operator \\
display; \\
allows \\
visualization \\
of message \\
traffic
\end{tabular} & \\
\hline
\end{tabular}




\section{APPENDIX G: TVA TESTING (JULY 2009)}

\section{Test Goals}

There were four goals (tests) defined for this phase of the Lemnos Interoperable Security Project architecture testing:

(1) Establish a baseline connection between all vendor participants through the Cisco switch;

(2) Determine the ability to establish multi-vendor interoperability within a power provider utility process control laboratory test environment;

(3) Determine the level of security that could be established within this interoperable system;

(4) Determine the capability of the differing systems to produce and transmit Syslog messages to a common Syslog file storage.

\section{Test Participants \& Equipment}

In addition to the Lemnos primary partners, Sandia National Laboratories (SNL) and Schweitzer Engineering Laboratories (SEL), four other vendor organizations participated in this phase of the Lemnos project testing: (1) Garrettcom, Inc., (2) n-Dimension, (3) Phoenix Contact, Inc., and (4) Industrial Defender, Inc. In the discussion to follow, a random assignment of vendor identifications has been assigned to each of the participants.

The specific vendor equipment systems that were used in this testing included:

- Sandia Lemnos/OPSAID Reference Architecture Prototype System (field \& system units)

- Schweitzer SEL 3620 Ethernet Security Gateway

- n-Dimension nPlatform 340

- Garrettcom Magnum DX900

- Industrial Defender ESP (Electronic Security Perimeter)

- Phoenix Contact MGuard RS

- Industrial Defender SEM (Security Event Manager) use in test for Syslog files

A Cisco 2950 Switch was used throughout the testing for IP addressing of each of the equipment units included. After core goal testing was completed, additional testing was conducted utilizing a Cisco ASA 5520 Adaptive Security Appliance.

A diagram of the test network is shown in Figure 1. The description of the test units are simply identified as Units A-E, related to each of the five vendor organizations participating in the tests. In this system, the SNL-1 field unit served as both a standard control system unit and a gateway between the vendors and the Syslog file system (SNL-2) and the Industrial Defender Syslog file system (SEM). The "hosts" identified were represented by each vendor's representative laptop computer for configuration definitions and view into message traffic \& diagnostics throughout the testing.

\section{Test Procedures \& Results}

The first step in conducting the tests was for each vendor to establish a Virtual Private Network (VPN) tunnel with Sandia and with each other. It was impressive that within two hours of the simple start of all units being connected to the Cisco 2950 and powering up all the equipment that 14 VPN tunnels for interoperability had been established (see Figure 2). One tunnel between two vendors (C \& E) was not completed because one vendor had implemented DH Group 2 while the other had implemented DH Group 5 for IKEv1. This problem could be overcome by the inclusion of additional software along with possible dynamic configuration capability that would permit more than one group being implemented or 
by "standardizing" on a single specific DH group. This highlights one of the problems of vendors needing to meet the expectations of their customers' needs and defining hard configurations.

The next set of tests conducted addressed authentication between systems and the logging of Syslog messages. The tests began with each of the systems establishing communication with each other by using shared password keys (PSK) and that was accomplished.

During the testing, all vendor units were able to send Syslog messages to both the Sandia-2 unit Syslog files and the Industrial Defender SEM. All Syslog message traffic was also viewed by all participants, shown in Figure 3. Syslog is typically used for computer system management and security auditing. For example, authentication failures would be reported across the control system through the Syslog messages. Furthermore, Syslog is supported by a wide variety of devices and receivers across multiple platforms and through the Lemnos tests, Syslog can be used to integrate log data from many different types of systems into a central repository. Both interoperability and security are improved through the use of Syslog.

A second step was to utilize security certificates signed by a certificated authority (CA). The established links shown in Figure 4 were a combination of self-signed certificates and certificates authenticated by a CA (the Sandia system can provide that authentication for this testing).

Near the end of the test period, a Cisco Adaptive Security Appliance (ASA) 5520 unit was configured into the network and three vendor participants in the test were able to established connections to that unit. Due to testing time restrictions, the other two vendors and Sandia did not make connections to the Cisco unit, although all three noted that they had made such connections in the past.

\section{Test Observations}

The key result from two days of testing by the six organizations represented at TVA is that it is possible to establish multi-vendor, secured interoperability and in a relatively short amount of time. This effort over the two day period included both actual floor testing time and several team meetings. Floor testing time totaled approximately 10 hours.

A major accomplishment of cooperation by all of the test participants was observed. All vendors worked around a table on their laptop computers connected to their respective control system (CS) units. These laptops provided the visualization, command, and configuration interfaces for the CS units. Discussions ranged from one-on-one to multiple-to-multiple. There was an excellent exchange of information about setting parameters and defining configurations throughout the entire testing process. Discussions were abundant about messages being sent/received, encryption codes being used, and parameters that were being used in assisting each other to establish interoperability. The cooperation among all participants was outstanding.

Nearly all of the testing procedures and steps required for the success of this testing were done in parallel. This permitted each of the participants to move ahead with next test stages as they completed each stage, permitting multi-way establishment of each level of interoperability and security testing.

\begin{tabular}{|c|c|c|c|c|c|c|}
\hline & \multicolumn{5}{|c|}{ Vendor Settings for Interoperability Testing - July 28-29, 2009} & \multirow[b]{2}{*}{ Sandia } \\
\hline Vendor & A & B & C & D & E & \\
\hline Parameter & & & & & & \\
\hline $\begin{array}{l}\text { P1 } \\
\text { Encryption }\end{array}$ & $\begin{array}{l}\text { 3DES, } \\
\text { AES- } \\
128 / 256\end{array}$ & $\begin{array}{l}\text { DES, 3DES, } \\
\text { AES- } \\
\text { 128/192/256 }\end{array}$ & $\begin{array}{l}\text { DES, } \\
\text { 3DES, } \\
\text { AES-128 }\end{array}$ & $\begin{array}{l}\text { DES, 3DES, } \\
\text { AES- } \\
\text { 128/192/256 }\end{array}$ & AES-128 & $\begin{array}{l}\text { DES, 3DES, } \\
\text { AES- } \\
\text { 128/192/256 }\end{array}$ \\
\hline P1 Hash & $\begin{array}{l}\text { MD5, } \\
\text { SHA1, } \\
\text { SHA2- } \\
\text { 256/512 }\end{array}$ & $\begin{array}{l}\text { MD-5, } \\
\text { SHA1, Auto }\end{array}$ & $\begin{array}{l}\text { MD-5, } \\
\text { SHA1 }\end{array}$ & $\begin{array}{l}\text { MD-5, SHA1, } \\
\text { Auto? }\end{array}$ & SHA1 & $\begin{array}{l}\text { MD-5, } \\
\text { SHA1/160, } \\
\text { SHA2- } \\
256 / 512 \\
\end{array}$ \\
\hline
\end{tabular}




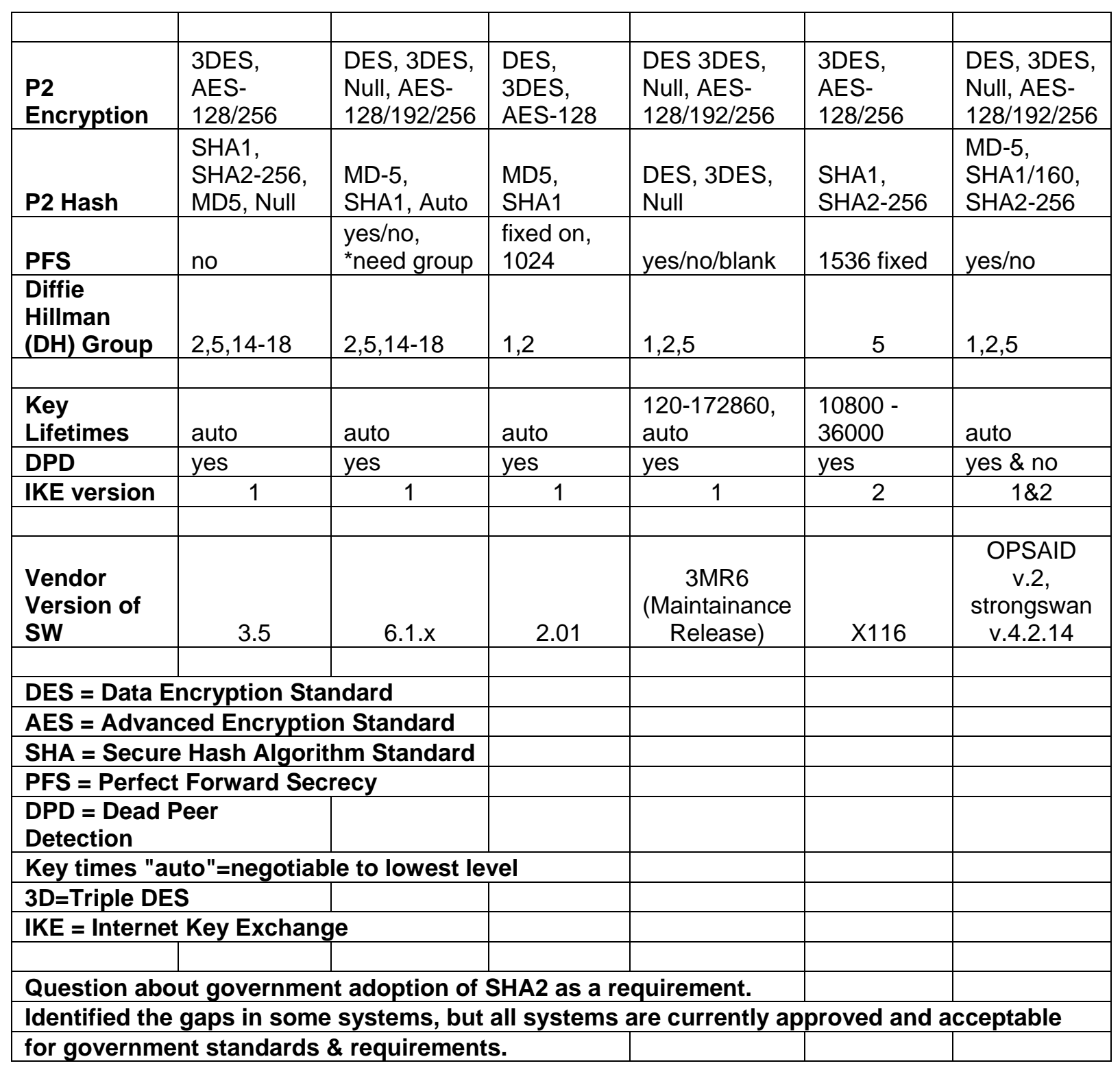

\section{Table G-1. Vendor Settings for Interoperability Testing}

The entire test team met and defined the range of parameters and capabilities included with each of their respective systems which are summarized above in Table 1. From the discussions that ensued as a part of the creation of this cross-reference table, gaps were identified for different vendors and tasking was taken back to their respective organizations for implementation.

\section{Conclusions}

Through the cooperative trial and error exercises with each of the systems, this testing demonstrated that it is possible to establish a baseline level of common configuration parameters and settings for interoperability and security between multiple vendors and that it has been demonstrated. It became evident that the efforts to establish secured interoperability by experts is a necessary step towards creating the information needed for utility companies to install multi-vendor supplied equipments for secured interoperability. There are areas where each vendor needs to pay some attention in looking ahead for the next year. 
The take-away from this set of testing and demonstration is that a major step has been taken to provide the information necessary to be shared amongst vendors and with utility companies for the establishment of secured interoperability. 


\section{APPENDIX H: EPRI TESTING (2011)}

Within the EPRI Smart Grid Substation Labs in Knoxville, TN, A basic testing architecture was created as shown in Figure 2-2 which supported testing of the IPSec, SSH, and LDAP Interoperable Configuration Profiles.

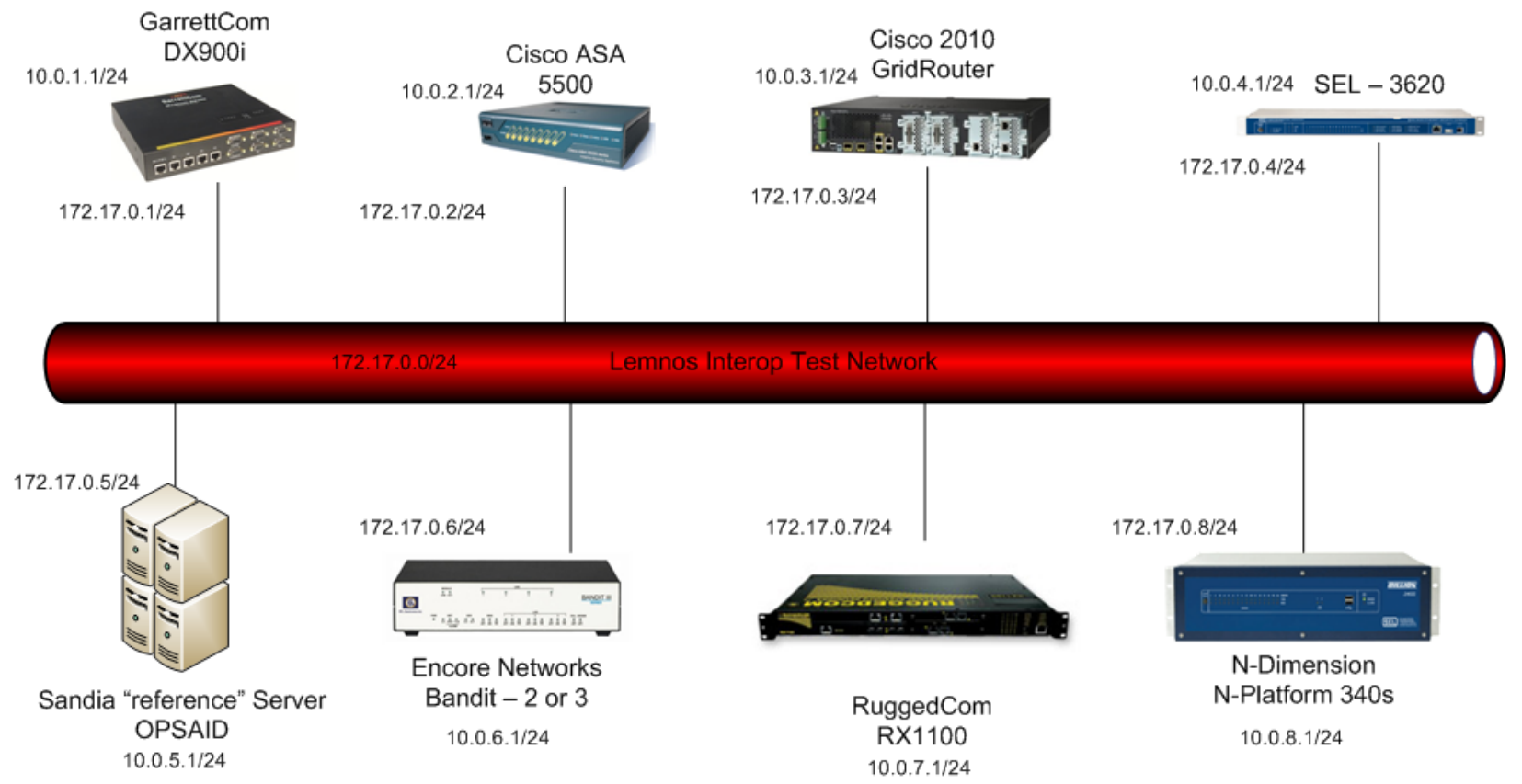

Figure H-1

EPRI Smart Grid Substation Labs - Lemnos Test Architecture 



\section{APPENDIX I: $\quad$ DEFINITIONSIACRONYMS}

AES - Advanced Encryption Standard (AES) is a symmetric-key encryption standard adopted by the U.S. government.

CA - Certificate Authority

IETF - Internet Engineering Task Force

IPsec - Internet Protocol Security (IPsec) is a protocol suite, defined by IETF RFC 4301 for securing Internet Protocol (IP) communications by authenticating and encrypting each IP packet of a communication session.

LDAP - Lightweight Directory Access Protocol (LDAP) is an application protocol for querying and modifying data of directory services implemented in Internet Protocol (IP) networks

RFC - Request for Comment

SSH - Secure Shell or SSH is a network protocol that allows data to be exchanged using a secure channel between two networked devices.

Syslog - Syslog is a standard, defined by IETF RFC 5424/3164, for logging program messages.

TLS - Transport Layer Security (TLS) is a cryptographic protocol that provides security for communications over networks. TLS encrypts the segments of network connections at the Application Layer to ensure secure end-to-end transit at the Transport Layer.

X.509 - X.509 is a Telecommunication Standardization Sector (ITU-T) standard for a public key infrastructure $(\mathrm{PKI})$ 